\title{
WestVirginiaUniversity
}

THE RESEARCH REPOSITORY @ WVU

Graduate Theses, Dissertations, and Problem Reports

2015

\section{Piece-wise Linear Support Vector Machine to Classify Distributed Data}

Nafiseh Salmani Niyasar

Follow this and additional works at: https://researchrepository.wvu.edu/etd

\section{Recommended Citation}

Salmani Niyasar, Nafiseh, "Piece-wise Linear Support Vector Machine to Classify Distributed Data" (2015). Graduate Theses, Dissertations, and Problem Reports. 7124.

https://researchrepository.wvu.edu/etd/7124

This Thesis is protected by copyright and/or related rights. It has been brought to you by the The Research Repository @ WVU with permission from the rights-holder(s). You are free to use this Thesis in any way that is permitted by the copyright and related rights legislation that applies to your use. For other uses you must obtain permission from the rights-holder(s) directly, unless additional rights are indicated by a Creative Commons license in the record and/ or on the work itself. This Thesis has been accepted for inclusion in WVU Graduate Theses, Dissertations, and Problem Reports collection by an authorized administrator of The Research Repository @ WVU. For more information, please contact researchrepository@mail.wvu.edu. 


\title{
Piece-wise Linear Support Vector Machine to Classify Distributed Data
}

\author{
Nafiseh Salmani Niyasar \\ Thesis submitted to the \\ College of Engineering and Mineral Resources \\ at West Virginia University \\ in partial fulfillment of the requirements \\ for the degree of \\ Master of Science \\ in \\ Computer Science \\ Afzel Noore, Ph.D., Chair \\ Yaser P. Fallah, Ph.D. \\ Xin Li, Ph.D.
}

Lane Department of Computer Science and Electrical Engineering

$$
\text { Morgantown, West Virginia }
$$

2014

Keywords: Support Vector Machine; Classification; Large Dataset;

Supervised Clustering; Distributed Classification

(C) 2014 Nafiseh Salmani Niyasar 


\section{Abstract \\ Piece-wise Linear Support Vector Machine to Classify Distributed Data}

\section{Nafiseh Salmani Niyasar}

Support Vector Machines (SVMs) is a popular and highly performing classification model with a very good generalization ability for many classification applications. This method uses kernels to classify data that are not linearly separable. The added complexity of the kernels that map data to a higher dimensional space degrade the SVM classifier performance when dealing with large datasets. Moreover, classifying a dataset by choosing an appropriate kernel and finding the best set of its parameters is challenging. Failing in this process can easily cause to an overfit problem.

In this thesis we propose the Piece-wise Linear SVM (PWLSVM) using MagKmeans clustering to address the complexity and computational cost of SVMs. We use a linear SVM to overcome the complexity of dealing with the kernels, and a MagKmeans clustering to cluster the data into balanced groups. MagKmeans which is a supervised technique clusters equal number of each class in one group. It ensures that a linear SVM has balanced training samples for each class and can attain an accurate model.

The detailed mathematical formulation and modeling of the proposed Distributed MagKmeans (D-MagKmeans) is presented. The algorithm uses a Distributed MagKmeans clustering approach to transform the PWLSVM to Distributed Piece-wise Linear SVM (D-PWLSVM). The proposed D-MagKmeans clustering approach makes the MagKmeans clustering work in distributed network by only passing the centroid of each cluster in one node only to its one-hop neighbors. This feature of the D-Magkmeans makes our approach appropriate for a distributed processing and decision making while maximizing privacy and minimizing the communication overload.

The proposed algorithm was validated using four datasets in terms of dimensions on the features and the number of samples. Pima Indian Diabetes, with 768 samples and 8 features, is the smallest dataset of the four. We also examined Abalone, with 4177 samples and 8 features, Waveform, with 5000 samples and 22 features, and EHarmony, with over half million samples and 116 features. The results reveal that a reasonable trade-off is required when dealing with a large dataset. The results also illustrate that PWLSVM and D-PWLSVM outperform SVMs on a relatively large dataset, such as EHarmony, Abalone, and Waveform. 


\section{Acknowledgments}

I wish to acknowledge the help and support of my adviser Dr. Afzel Noore for his support of my Masters study and research, patience and guidance.

Beside my adviser, I would like to thank Dr. Yaser P. Fallah and Dr. Xin Li, my committee members for their encouragement, insightful words and questions during my defense session.

Last but not the least, I would like to thank my parents who always supported me throughout my life and also my sisters especially Kimia Salmani for her unconditional love and support. 


\section{Contents}

1 Introduction 1

1.1 An overview on Support Vector Machine . . . . . . . . . . . . . . . 1

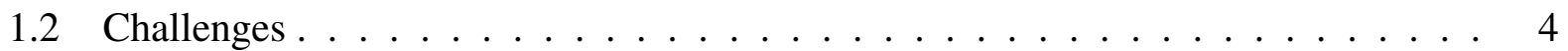

1.2.1 SVMs and Kernel Selection _. . . . . . . . . . . . . . . 4

1.2.2 SVMs and Large Datasets . . . . . . . . . . . . . . . . . . . . . 5

1.3 Research Objectives . . . . . . . . . . . . . . . . . . . 7

1.4 Contribution of the Thesis . . . . . . . . . . . . . . . . 7

1.5 Thesis Outline . . . . . . . . . . . . . . . . . . 8

2 Piece-wise Linear SVM 9

2.1 MagKmeans . . . . . . . . . . . . . . . . . . . . . 9

2.2 Piece-wise Linear SVM . . . . . . . . . . . . . . . . . . . . . . . . . . . . . . . . . . . . . . . .

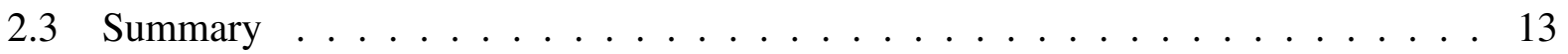

3 Distributed Piece-wise Linear SVM using a Distributed MagKmeans 14

3.1 Distributed MagKmeans . . . . . . . . . . . . . . . . . 15

3.1 .1 Formulation . . . . . . . . . . . . . . . 15

3.1.2 Proposed Distributed MagKmeans (D-MagKmeans) Algorithm . . . . . . 20

3.2 Tests and Results . . . . . . . . . . . . . . . . . . . . . . 21

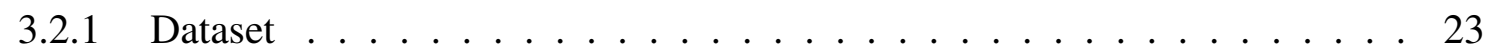

3.3 Distributed Piece-wise Linear SVM Using D-MagKmeans (D-PWLSVM) . . . . . 26

3.3 .1 Dataset . . . . . . . . . . . . . . . 27

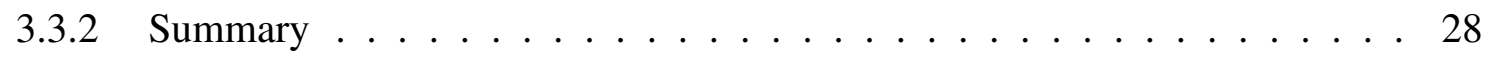

4 Experiments and Results 29

4.1 Datasets . . . . . . . . . . . . . . . . . . . . . . . . 29

4.1.1 Pima Indians Diabetes Dataset . . . . . . . . . . . . . . 29

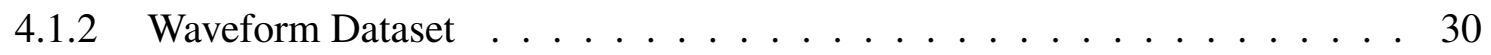

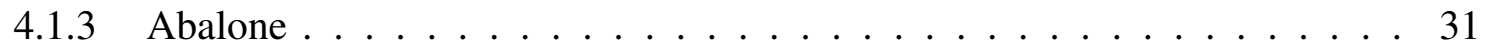

4.1 .4 EHarmony Dataset . . . . . . . . . . . . . . . . 32

4.2 Experimental Results . . . . . . . . . . . . . . . . . . . . . 32

4.2.1 Pima Indian Diabetes . . . . . . . . . . . . . . . . . . . . . . . . . . . . . . . 33

4.2.2 Waveform Dataset . . . . . . . . . . . . . . . 35 
4.2.3 Abalone Dataset . . . . . . . . . . . . . . . . . . . . . 37

4.2 .4 EHarmony . . . . . . . . . . . . . . . . . . . . . . 39

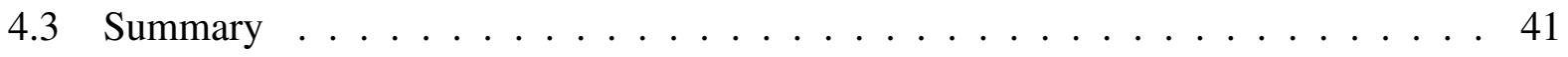

5 Conclusion and Future Work $4 \mathbf{4 2}$

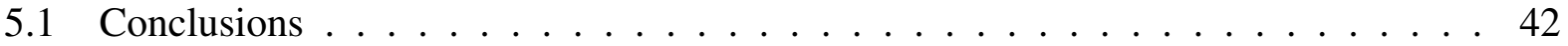

5.2 Future Work . . . . . . . . . . . . . . . . . . . . 43 


\section{List of Figures}

1.1 A view of SVM margin $\ldots \ldots \ldots \ldots \ldots$

3.1 An example of a 5-node network with the corresponding parameters $\ldots \ldots 16$

3.2 A view of D-MagKmeans algorithm . . . . . . . . . . . . . . . . 21

3.3 Results of applying the D-MagKmeans on a 3-node network (each color reprsents

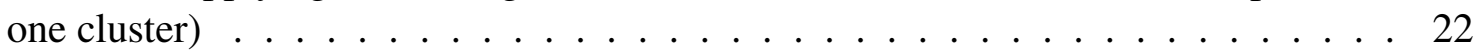

3.4 A view of a 3-node network connectivity . . . . . . . . . . . . . . 23

3.5 Clustering cost comparison of 500 runs each of D-MagKmeans and MagKmeans

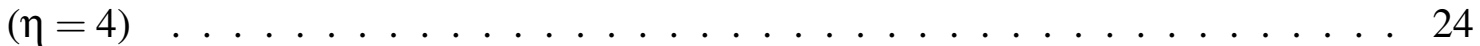

3.6 Training process of D-MagKmeans for different $\eta$, representing the cost of clustering, per iteration . . . . . . . . . . . . . . . 25

3.7 Clustering results after applying D-MagKmeans . . . . . . . . . . . . . . 26

3.8 Simple example of an D-PWLSVM . . . . . . . . . . . . . . . . 27

3.9 Results of the D-PWLSVM on randomly generated data $\ldots \ldots \ldots 28$

4.1 Error comparison between D-PWLSVM, PWLSVM, and classic SVM on the Diabetes dataset . . . . . . . . . . . . . . . . . . . . . . . . 34

4.2 Training time comparison between D-PWLSVM, PWLSVM, and classic SVMs on the Diabetes dataset . . . . . . . . . . . . . . . . . . . . . . . . 34

4.3 Error comparison between the D-PWLSVM, PWLSVM and, classic SVMs on the Waveform dataset . . . . . . . . . . . . . . . . . . . . . 36

4.4 Training time comparison between D-PWLSVM, PWLSVM, and classic SVMs on the Waveform dataset . . . . . . . . . . . . . . . . . . . . 36

4.5 Error comparison between the D-PWLSVM, PWLSVM, and the classic SVMs on the Abalone dataset . . . . . . . . . . . . . . . . . . . . . . . 38

4.6 Training time comparison between the D-PWLSVM, PWLSVM, and the classic SVMs on the Abalone dataset . . . . . . . . . . . . . . . . . . . . . 38

4.7 Error comparison between D-PWLSVM, PWLSVM, and the classic SVMs on the EHarmony dataset . . . . . . . . . . . . . . . . . . . . . . 4 40

4.8 Training time comparison between the D-PWLSVM, PWLSVM, and the classic SVMs on the EHarmony dataset . . . . . . . . . . . . . . . . . . . 40 


\section{List of Tables}

3.1 Cost comparison of D-MagKmeans and centralized MagKmeans . . . . . . . . 24

4.1 Performance comparison (error and training time) between classic SVM, PWLSVM, and D-PWLSVM on the Diabetes dataset . . . . . . . . . . . . . . 33

4.2 Performance comparison (error and training time) between the classic SVM, PWLSVM, and D-PWLSVM on the Waveform dataset . . . . . . . . . . . . . . . . . 35

4.3 Comparison of results between the classic SVM, PWLSVM, and D-PWLSVM on the Abalone dataset . . . . . . . . . . . . . . . . . . . . . . . 37

4.4 Comparison of the results between classic SVM, PWLSVM, and D-PWLSVM on the EHarmony dataset . . . . . . . . . . . . . . . . . . 41 


\section{Abbreviations}

D-MagKmeans: Distributed MagKmeans

D-PWLSVM: Distributed Piece-wise Linear SVM

GSVM: Growing SVM

LP: Linear Programming

LSVM: Linear SVM

PSVM: Profile SVM

PWLSVM: Piece-wise Linear SVM

RBF: Radial Basis Function

SMO: Sequential Minimal Optimizer

STD: Standard Deviation

SV: Support Vector

SVM: Support Vector Machine

UCI: University of California, Irvine

WLS-SVM: Weighted Least Mean Square SVM 


\section{Chapter 1}

\section{Introduction}

\subsection{An overview on Support Vector Machine}

The Support Vector Machine (SVM) is an accurate and popular classifier in several different research fields $[5,6,31]$; however, the algorithm is best suited for small amounts of training data and works exceptionally slowly with non-linear separable training data. An SVM classifier tries to find the maximum-margin classifier, based on the centrally available data. To find a classifier in high dimensional data, which are not linearly separable, a pre-defined kernel function is used. In these circumstances, the classifier accuracy is highly dependent on finding an appropriate kernel and its optimal parameters. Ideally the non-linear training data can be mapped to a higher dimension in which the data are linearly separable.

SVM classifier tries to find a hyperplane, $w^{T} x-b=0$, to separate a set of positively and negatively labeled training data space (labeled as -1 and +1 ). Note that $w$ is a vector orthogonal to the hyperplane and $b$ is the bias. This classifier maximizes the margins, while trying to minimize the classification error by solving a non-linear problem (Eq. 1.1). The margin is defined by the 
distance of two other parallel hyperplanes, with a decision surface where $\left|w^{T} x_{i}-b\right|=1$.

$$
\text { Minimize }\left(\frac{1}{2}\right) w^{T} w
$$

$$
\text { Subject to } y_{i}\left(w^{T} x_{i}-b\right) \geq 1, i=1, \ldots, N
$$

Eq. 1.2 is the dual form of Eq. 1.1

$$
\begin{aligned}
& \text { Maximize }-\left(\frac{1}{2}\right) \sum_{i, j=1}^{N} \alpha_{i} y_{i} y_{j} x_{i}^{T} x_{j} \alpha_{j}+\sum_{i=1}^{N} \alpha_{i} \\
& \text { Subject to } \alpha_{i} \geq 1, i=1, \ldots, N \text { and } Y^{T} \alpha=0
\end{aligned}
$$

where, $\alpha$ is a vector with $N$ number of data and $Y$ represents the class labels.

Equation 1.2 is a quadratic programming problem. At the optimum solution, the Support Vectors (SVs) are placed in the margin hyperplane. This is only true if the data is linearly separable. For data which are not linearly separable, a set of non-negative slack variables $(\xi \geq 0)$ are used to measure the degree of the misclassification of the data $x_{i}$. Therefore, the optimization problem changes to find a trade-off between a large margin and the smaller error penalty (Eq. 1.3 and Fig. $1.1)$.

$$
\begin{gathered}
\text { Minimize }\left(\frac{1}{2}\right) w^{T} w+\gamma \sum_{i=1}^{n} \xi_{i} \\
\text { Subject to } y_{i}\left(w^{T} x_{i}-b\right) \geq 1-\xi_{i}, i=1, \ldots, N
\end{gathered}
$$

The dual for Eq. 1.3 is in the form of Eq. 1.4.

$$
\begin{gathered}
\text { Maximize }-\left(\frac{1}{2}\right) \sum_{i, j=1}^{N} \alpha_{i} y_{i} y_{j} x_{i}^{T} x_{j} \alpha_{j}+\sum_{i=1}^{N} \alpha_{i} \\
\text { Subject to } \gamma \geq \alpha_{i} \geq 1, i=1, \ldots, N \text { and } Y^{T} \alpha=0
\end{gathered}
$$




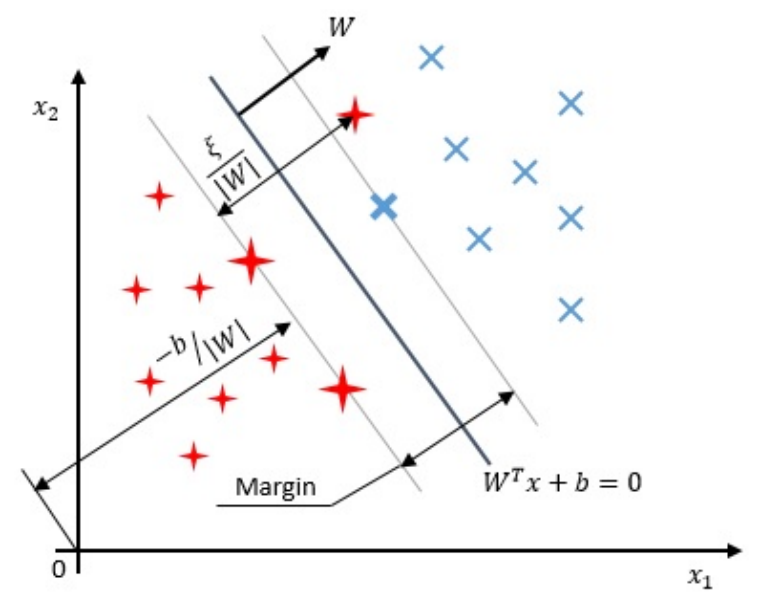

Figure 1.1: A view of SVM margin

Scalar $\gamma$ is a tuning parameter. Choosing a proper value for $\gamma$ can be important to reduce the error rate. Using the slack variable, the SVs can either lie between the margin hyperplanes or be misclassified. If a decision surface could not be achieved in a linear form, a non-linear kernel function can be used. The kernel function $\phi(x): R^{m} \rightarrow R^{n}$ is used to map $m$ dimensional training data into $n$ dimensions $(n>m)$.

$$
\begin{gathered}
\text { Maximize }-\left(\frac{1}{2}\right) \sum_{i, j=1}^{N} \alpha_{i} y_{i} y_{j} \phi\left(x_{i}\right)^{T} \phi\left(x_{j}\right) \alpha_{j}+\sum_{i=1}^{N} \alpha_{i} \\
\text { Subject to } \gamma \geq \alpha_{i} \geq 1, i=1, \ldots, N \text { and } Y^{T} \alpha=0
\end{gathered}
$$

Equation 1.5 can be re-written as Eq. 1.6.

$$
\begin{aligned}
& \text { Maximize }-\left(\frac{1}{2}\right) \sum_{i, j=1}^{N} \alpha_{i} \alpha_{j} y_{i} y_{j} F\left(x_{i}, x_{j}\right)+\sum_{i=1}^{N} \alpha_{i} \\
& \text { Subject to } \gamma \geq \alpha_{i} \geq 1, i=1, \ldots, N \text { and } Y^{T} \alpha=0
\end{aligned}
$$

In Eq. 1.6, $\phi\left(x_{i}\right)^{T} \phi\left(x_{j}\right)$ is replaced by a kernel function $F\left(x_{i}, x_{j}\right)=k_{i j}$, where $k$ is called the 
kernel matrix. This method is called the "Kernel trick". SVM maps the training data into a higher dimensional data using a kernel function in order to make the data linearly separable. Equation 1.6 has only one optimum solution if the kernel matrix is positive definite; a symmetric $n \times n$ real matrix $M$ is said to be "positive definite" if $z^{T} M z$ is positive for every non-zero column vector $z$ of n real numbers.

\subsection{Challenges}

All classification techniques have advantages and disadvantages. These are more or less important according to the data the selected technique is performed on. SVM can be a useful tool when the data are not regularly distributed or have an unknown distribution [1]. However, when there are a huge quantity of data, the SVM faces many challenges. Two of these challenges are as follows:

\subsubsection{SVMs and Kernel Selection}

By introducing the kernel trick, SVMs gain the ability to classify data which are not linearly separable. However there is almost no guide to help find an appropriate kernel function for a specific application. Kernel selection always requires knowledge about the problem in advance [24]. There is a variety of research on different kernels, including the radial basis kernel [5, 14], polynomial kernel functions [23,27], and other kernels [7, 15].

Finding a kernel is always a challenge to attain a better classifier with a minimum error. Complex kernel functions can lead to a problem in which a decision surface closely fits the training data, such that it is unable to classify new test data well. After a certain point, adding new features and increasing the dimensionality of the problem would actually degrade the performance of a classifier. This effect is referred to as the "Curse of Dimensionality". The direct result of this effect is "overfitting" which is when the classifier learns the specific instances and exceptions of the training data in such a way that the classifier would fail more frequently on real-world data 
from an unseen dataset.

Moreover, classification using non-linear kernel functions can be expensive if $\mathrm{n}$ (the dimensionality of the mapped data) is very high. Yuan et al. in 2012 [30] compared linear SVM and non-linear SVM. They found that although non-linear SVM has better accuracy, in general, as compared to linear SVM, when using large scale data, the accuracies of both methods are almost identical. Considering testing and training time, the linear SVM is much faster than the non-linear SVM [30].

\subsubsection{SVMs and Large Datasets}

SVMs use a centralized approach. As such, they are mostly suited for small amounts of training data and work exceptionally slow when they are applied to large datasets. Given $m$ training data, the training complexity of quadratic programming problem is $O\left(m^{3}\right)$ and its space complexity is $O\left(n^{2}\right)$ $[2,9,29]$. To address this problem, several studies have focused on large dataset classifications that were categorized into four groups such as greedy approximations, sampling, matrix decomposition, and methods that scale down the training samples before employing the SVM.

An incremental approach was proposed for the first time in 1999 by Ahmed Syed et al. [26]. In this approach, training data splits into different partitions called $T R_{i} . S V M_{1}$ is trained by using $T R_{1}$, then $S V M_{2}$ is trained using $T R_{2}$ and the support vectors achieved using $T R_{1}\left(S V_{1}\right)$, and so on. Shilton and Palaniswami proposed another approach for incremental training in 2005 [25]. They used a warm start and found a solution for new arrival training data using the old solution obtained in the previous iterations. They used an active set approach to solve the SVM quadratic problem.

Sequential minimal optimization (SMO) [22] was also proposed in 1999. This technique breaks the large quadratic problem into a series of small active sets, each of which carries only two variables. The Boost-SMO approach has been presented for speeding up SMO [21].

The Weighted Least Mean Square SVM (WLS-SVM) [5] is an iterative training algorithm using the Weighted Least Mean Square. Although the performance of the SVM has been shown to 
degrade using this method, it has helped to reduce the complexity and size of SVs [19].

Parrado-Hernandez et al. in 2003, proposed a modification of the use of SVM with the WLSSVM algorithm called Growing SVM (GSVM) [20], which selects SVs more precisely. The GSVM algorithm starts with an $M$ number of training samples $(M / 2$ per class) and adds new centroids at each step to update the weights.

The boosting method was used to overcome the complexity and time computational costs caused by a Principal Component Analysis or clustering training samples. GSVM considers a pool of candidates to count as centroids and adds them to the machine in each step. At step " $n$ ", the machine will update from step " $n-1$ "; as such, there is no need to train the machine from the beginning. Additionally, non-support vectors are eliminated at each step to speed up the training stage.

In 2004, Wen and Lu proposed a cascade method for reducing the training time and the number of SVs, by dividing the training data into different groups and applying an SVM on each partition, with the SVs at each step transferring to the next SVM [28]. Hence, the number of training samples for each SVM is smaller than the original dataset and each SVM can run simultaneously, thereby making the process faster. The drawback to this approach is that the obtained SVs in each group are not the same as the SVs of when applying SVM on all of the data together; therefore, the classifier performance is reduced.

Several studies have focused on the parallel design of SVM [5,6,17,24], while others attempted to use clustering techniques and convex hulls to address the SVMs problems [8,9]. In addition, some methods try to reduce the number of SVs [12,31] which could reduce accuracy.

The SVMs using Adaptive Clustering, tries to speed up the training time with the clustering. This is accomplished by applying SVM on potential SVs rather than the entire training dataset [4]. Boley et al. certified that Adaptive Clustering was a faster algorithm in comparison to the SMO. SVMs, based on Fuzzy Clustering, have been shown to achieve a faster approach for large datasets in comparison to traditional SVMs [9]. 
In 2006, a distributed SVM was proposed [18]. Using this method each node trains on its own training data using the Growing SVM (GSVM) and then transfers their centroids to the rest. In 2010, Pedro A. Forero introduced a consensus-based distributed SVM [13]. Using this method, a unique model was obtained across the entire network, while providing security of data in each of the computer networks. All of these approaches attempt to address one of the problems at a time; as such, they all have their own advantages and disadvantages.

\subsection{Research Objectives}

In this thesis our focus is to develop an approach to speed-up the SVM training process, while specifically addressing the problem of choosing appropriate kernel for large datasets.

- Using Piece-wise Linear SVM (PWLSVM), we introduce a faster approach, as compared to SVMs. The use of a linear SVM eliminates the complexity of the kernels and the difficulty of finding an appropriate kernel and optimal parameters.

- Proposing the Distributed Piece-wise Linear SVM (D-PWLSVM) to address the centralization problem and offer a solution to the environment of privacy consideration.

\subsection{Contribution of the Thesis}

In this thesis work, we have made an attempt to address the complexity and computational cost of SVM by proposing the combined Piece-wise Linear SVM (PWLSVM) method. In this approach linear SVM is used to overcome the complexity of using kernels and MagKmeans clustering is used to cluster data into balanced groups.

We also expanded PWLSVM on centralized data to distributed data. In order to do that we developed Distributed MagKmeans clustering (D-MagKmeans). Using D-MagKmeans, only centroids of each clusters are passed between distributed nodes. This feature of MagKmeans makes 
our approach appropriate when the data source is distributed and when privacy of data and reliable cost-effective communication are major concerns.

\subsection{Thesis Outline}

Chapters 2 and 3 explain the centralized and distributed piece-wise linear SVM using MagKmeans. The experimental results are discussed in Chapter 4. Chapter 5 includes the conclusions, as well as recommendations for further work. 


\section{Chapter 2}

\section{Piece-wise Linear SVM}

SVM, are proven to be highly performing classifiers. However, in terms of classification speed, they are very slow when it comes to the classification of large datasets. Consequently, in this chapter, we introduce a new approach "Piece-wise Linear SVM" which classifies large datasets at a much faster rate than general SVM classifiers. The primary goal of this study is to present an approach that performs faster and keeps the classifier accuracy as close as possible to that of the classic SVMs. To accomplish this goal, we use MagKmeans to cluster the data into multiple groups and a linear SVM to find the classifier model. The results reveal that this approach leads to a faster classifier with acceptable accuracy, in comparison to the classic methods on large datasets.

The rest of this chapter is organized as follows. Section 2.1 describes the MagKmeans Clustering. The Piece-wise Linear SVM is described in 2.2 and this chapter ends with a summary in Section 2.3.

\subsection{MagKmeans}

The MagKmeans is a supervised algorithm developed by Haibin Cheng et al. in 2010 [11]. This algorithm clusters pre-labeled data into multiple groups, each of which includes a balanced number 
of class members. Profile SVM (PSVM), presented in [11], is a localized algorithm that attempts to find the local optimum solution each time a new group of test data is added. In this approach, the MagKmeans clusters by using a similarity matrix between the training and test data. Similarity matrix $\Sigma$ is defined as an $n \times m$ matrix in which the value in the $i^{t h}$ row and $j^{\text {th }}$ column represents similarity between the training sample $\left(x_{i}\right)$ and the test sample $\left(\bar{x}_{j}\right)$. Given $D$, a set of $n$ training data, Eq. 2.1 illustrates the objective function of the MagKmeans clustering.

$$
\min _{C, Z} \sum_{j=1}^{J} \sum_{i=1}^{n} Z_{i, j}\left\|X_{i}-C_{j}\right\|_{2}^{2}+R \sum_{j=1}^{J}\left|\sum_{i=1}^{n} Z_{i, j} Y_{i}\right|
$$

subject to:

$$
\begin{gathered}
0 \leq Z_{i, j} \leq 1, \forall i \in\{1, . ., n\}, j \in\{1, . ., J\} \\
\sum_{j=1}^{J} Z_{i, j}=1
\end{gathered}
$$

where, $X_{i}$ is the $i^{t h}$ row of the similarity matrix and $C_{j}$ is an $1 \times m$ row vector containing the centroids of the $j^{\text {th }}$ cluster. $R$ is a non-negative constant and $Y_{i}$ represents the class label of the $i^{t h}$ training sample. $Z_{i, j} \in[0,1]$ is the membership value of the $i^{\text {th }}$ training sample to the $j^{\text {th }}$ cluster and $J$ is the number of clusters.

The first part of the equation in Eq. 2.1 is the same as the objective function that appears in the Kmeans clustering. The second part tries to keep the class members balanced.

The main differences between this approach and the unsupervised clustering K-Means are twofolds: (1) the similarity matrix is being clustered, instead of the training samples; and (2) this algorithm considers the class distribution, as well as the similarity between the examples, such that all clusters will contain enough representative examples from the different classes.

In order to solve Eq. 2.1 and obtain the centroids of each cluster and the membership matrix $Z$ that can satisfy the objective function, $\left|\sum_{i=1}^{n} Z_{i, j} y_{i}\right|$ is replaced with slack variables $\tau_{j}$ where, $j=1, \ldots, J$. 


$$
\min _{Z, \tau} \sum_{i=1}^{n} \sum_{j=1}^{J} Z_{i, j}\left\|X_{i}-C_{j}\right\|_{2}^{2}+R \sum_{j=1}^{J} \tau_{j}
$$

subject to:

$$
\begin{gathered}
-\tau_{j} \leq \sum_{i=1}^{n} Z_{i, j} y_{i} \leq \tau_{j}, \forall j \in\{1, . ., J\} \\
\tau_{j} \geq 0,0 \leq Z_{i, j} \leq 1, \forall i \in\{1, . ., n\}, j \in\{1, \ldots, J\} \\
\sum_{j=1}^{J} Z_{i, j}=1, \forall i \in\{1, . ., n\}
\end{gathered}
$$

Eq. 2.2 can now be solved using linear programming methods. Algorithm 1 represents the MagKmeans clustering.

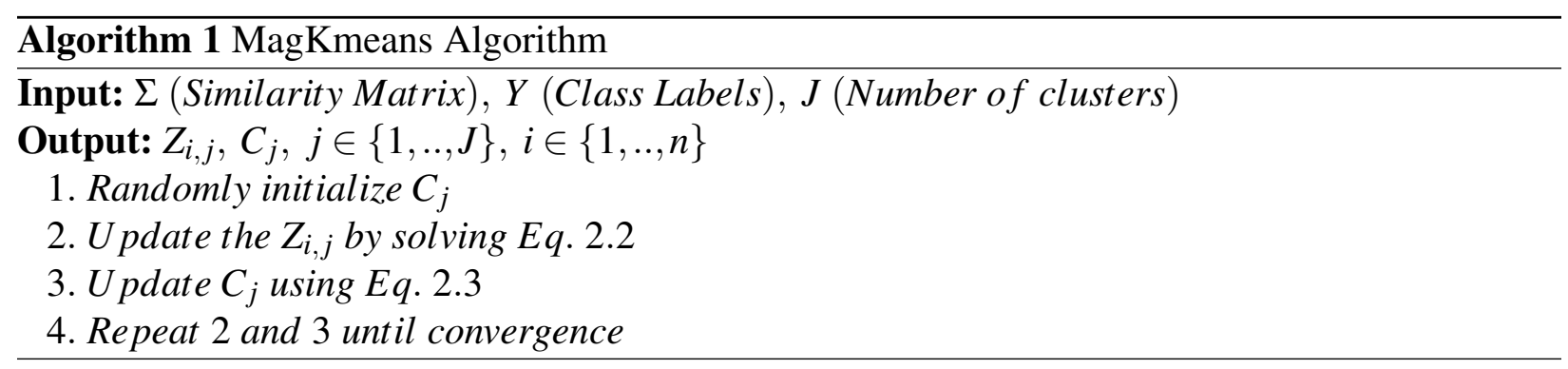

This approach is an iterative algorithm trying to find the membership matrix $Z$ using linear programming by fixing the cluster centroids at the beginning of each iteration. When the cluster memberships $\left(Z_{i, j}\right)$ are obtained, the centroids of each cluster $\left(C_{j}\right)$ can be updated using Eq. 2.3.

$$
C_{j}=\frac{\sum_{i=1}^{n} Z_{i, j} X_{i}}{\sum_{i=1}^{n} Z_{i, j}}
$$

The MagKmeans works on a single computer, using the entire dataset simultaneously (centralized). This algorithm needs both testing and training data, at the same time, to generate the similarity matrix and employ clustering. Every iterative step has to be performed when a new set of test data is used (localized). We used the MagKmeans in our approach to ensure that there was 
enough data from each class per cluster to obtain a better classifier when applying a linear SVM.

\subsection{Piece-wise Linear SVM}

The linear SVM (LSVM) performs better than the non-linear SVM, when it comes to large datasets [30]. However, this classifier is very slow. In this thesis, we introduce a Piece-wise Linear SVM (PWLSVM) to overcome this bottleneck. PWLSVM divides the data into smaller groups by using MagKmeans. Then, linear SVM is applied on each cluster to find the classifier models. Using MagKmeans ensures that each cluster has balanced information from both class distributions before applying a linear SVM separately to the clusters.

Since the MagKmeans clusters the similarity matrix between the test and training data, it incrementally approaches to the local optimum every time there is a new group of test data. Moreover, the similarity matrix can be huge for large datasets; as such, calculating the similarity matrix would be expensive in terms of time and memory storage.

We use training data, instead of the similarity matrix, as the input of the MagKmeans. Therefore, in Eq. 2.1, 2.2, and 2.3, $X_{i}$ represents the $i^{\text {th }}$ sample of the training data. Algorithm 2 summarizes the PWLSVM using the MagKmeans. The training data, classifier model and test data of the Linear SVM(LSVM) in the PWLSVM will subsequently be described. After applying the MagKmeans, members of each cluster are the former training samples for the LSVM. The LSVM is applied on members of each cluster separately.Based on the training data for each cluster, a decision boundary is determined to separate the two classes. When a new test data arrives, we first determine the appropriate cluster decision boundary based on the distance to cluster centroids. The decision boundary with the minimum distance is chosen for this purpose. 


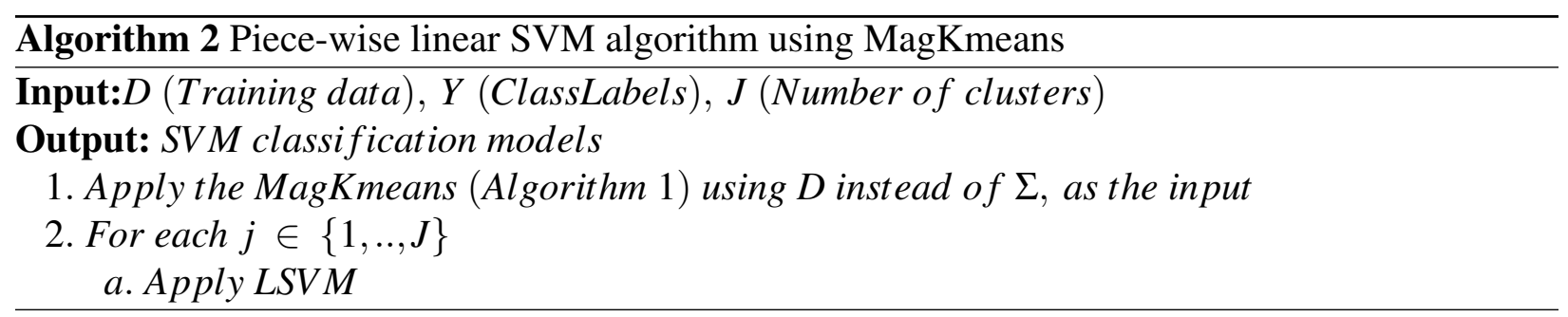

\subsection{Summary}

This chapter proposes a new PWLSVM approach using MagKmeans and a linear SVM. This approach is mostly suited for a centralized environment when dealing with large datasets. Algorithm 1 describes the MagKmeans algorithm. The proposed PWLSVM algorithm is described in Algorithm 2. 


\section{Chapter 3}

\section{Distributed Piece-wise Linear SVM using a Distributed MagKmeans}

Distributed learning solutions are mainly considered for applications in which having training data in a centralized unit is not feasible, for reasons such as privacy, cost of communication between network nodes, and large datasets. In this chapter, we propose a Distributed Piece-wise Linear SVM (D-PWLSVM) classifier model for the distributed networks. This chapter is organized as follows:

Section 3.1 proposes the Distributed MagKmeans (D-MagKmeans) formulation and model in detail. In order to illustrate how the distributed MagKmeans works on data, the results of applying the D-MagKmeans on a randomly generated dataset is discussed in Section 3.2. The Distributed Piece-wise Linear SVM steps are explained in Section 3.3. A summary of this chapter is presented in Section 3.4. 


\subsection{Distributed MagKmeans}

In this section we propose a method using a Lagrangian heuristic technique to apply the MagKmeans, a supervised clustering algorithm described in Chapter 2, in a distributed environment. In using this algorithm, data can be clustered in a distributed fashion while only the centroids of the clusters are transferred between the immediate neighbors of each node in a network. In what follows, this method will be mathematically proven.

\subsubsection{Formulation}

Having L number of distributed nodes in a network the MagKmeans objective function can be written as follows:

$$
\min _{C, Z} \sum_{l=1}^{L} \sum_{i=1}^{n_{l}} \sum_{j=1}^{J} Z_{l, i, j}\left\|X_{l, i}-C_{l, j}\right\|_{2}^{2}+R \sum_{l=1}^{L} \sum_{j=1}^{J}\left|\sum_{i=1}^{n_{l}} Z_{i, j} Y_{i}\right|
$$

Subject to:

$$
\begin{gathered}
0 \leq Z_{l, i, j} \leq 1, \forall i \in\left\{1, . ., n_{l}\right\}, j \in\{1, . ., J\}, l \in\{1, . ., L\} \\
\sum_{j=1}^{J} Z_{l, i, j}=1, \forall i \in\left\{1, . ., n_{l}\right\}, j \in\{1, . ., J\}, l \in\{1, . ., L\}
\end{gathered}
$$

where $n_{l}$ represents number of training data in node $l$. The term $\sum_{l=1}^{L}$ is added to the Eq. 2.1 (MagKmeans objective function), since our goal is to locate the center of each cluster (C) and the membership matrix (Z) in a way that minimizes Eq. 2.1 for all nodes in a network. Therefore, Eq. 2.1 can be rewritten in the form of Eq. 3.1 in a distributed environment. Moreover, two more constraints, listed in Eq. 3.2, need to be added to the objective function to assure that the center of each cluster in one node is equivalent to the center of same cluster in its adjacent nodes. Hence, assuming auxiliary variables $S_{l, p, j}$ Eq. 3.1 can be solved in a distributed manner.

$$
C_{l, j}=S_{l, p, j}, \forall l \in L, j \in J, p \in H_{l}
$$




$$
S_{l, p, j}=C_{p, j}, \forall l \in L, j \in J, p \in H_{l}
$$

where: $H_{l}$ stands for the nodes adjacent to the $l^{\text {th }}$ node. Fig 3.1 shows a network with its parameters.

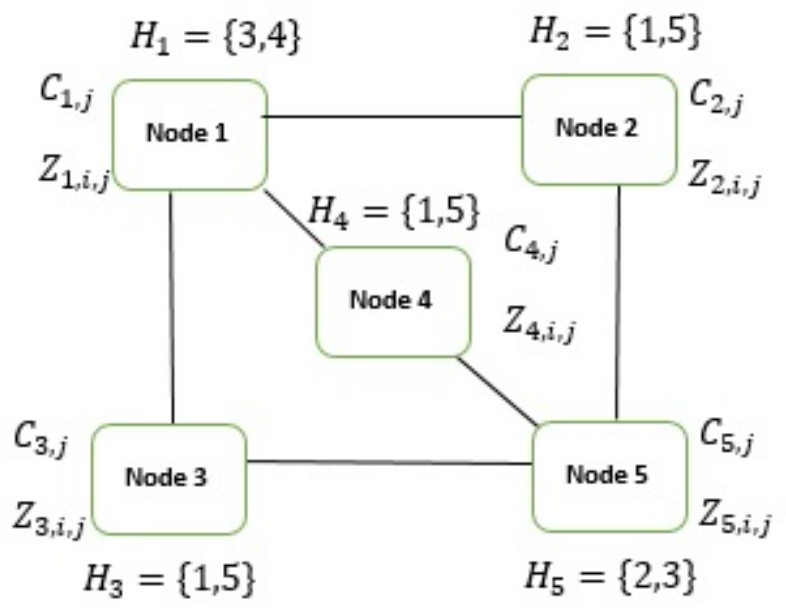

Figure 3.1: An example of a 5-node network with the corresponding parameters

To solve the constrained optimization problem of Eq. 3.1 with respect to the constraints in Eq. 3.2 in an optimal amount of time, the Augmented Lagrangian method [16] is used. Using this, a constrained optimization problem was replaced by several unconstrained problems, as follows:

$$
\begin{gathered}
F\left(Z_{l, i, j}, C_{l, j}, S_{l, p, j}, \beta_{l, p, j}, \gamma_{l, p, j}\right)= \\
\sum_{l=1}^{L} \sum_{i=1}^{n_{l}} \sum_{j=1}^{J} Z_{l, i, j}\left\|X_{l, i}-C_{l, j}\right\|_{2}^{2}+R \sum_{l=1}^{L} \sum_{j=1}^{J}\left|\sum_{i=1}^{n} Z_{l, i, j} Y_{l, i}\right| \\
+\sum_{l=1}^{L} \sum_{j=1}^{J} \sum_{p \in H_{l}} \beta_{l, p, j}\left(C_{l, j}-S_{l, p, j}\right)+\gamma_{l, p, j}\left(S_{l, p, j}-C_{p, j}\right) \\
+\frac{\eta}{2} \sum_{l=1}^{L} \sum_{j=1}^{J} \sum_{p \in H_{l}}\left\|C_{l, j}-S_{l, p, j}\right\|^{2}+\left\|S_{l, p, j}-C_{p, j}\right\|^{2}
\end{gathered}
$$


Subject to:

$$
\begin{gathered}
0 \leq Z_{l, i, j} \leq 1, \forall i \in\left\{1, . ., n_{l}\right\}, j \in\{1, . ., J\}, l \in\{1, . ., L\} \\
\sum_{j=1}^{J} Z_{l, i, j}=1, \forall i \in\left\{1, . ., n_{l}\right\}, l \in\{1, . ., L\}
\end{gathered}
$$

where: $\beta_{l, p, j}$ and $\gamma_{l, p, j}$ are the Lagrangian multipliers and $\eta$ is a positive scalar. The optimum solution for each variable in the Lagrangian function can be achieved iteratively, by assuming one variable at a time and solving the equation. Adding $\left\|S_{l, p, j}-C_{p, j}\right\|^{2}$ and $\left\|C_{l, j}-S_{l, p, j}\right\|^{2}$ as parts of the augmented equation help the objective function stay strictly convex in each step toward the solution. Therefore finding a unique optimal solution for each variable can be guaranteed.

$$
\begin{gathered}
Z_{l, i, j}(t+1)=\arg \min _{Z_{l, i, j}} F\left(Z_{l, i, j}, C_{l, j}(t), S_{l, p, j}(t), \beta_{l, p, j}(t), \gamma_{l, p, j}(t)\right) \\
S_{l, p, j}(t+1)=\arg \min _{S_{l, p, j}} F\left(Z_{l, i, j}(t+1), C_{l, j}(t+1), S_{l, p, j}, \beta_{l, p, j}(t), \gamma_{l, p, j}(t)\right) \\
C_{l, j}(t+1)=\arg \min _{C_{l, j}} F\left(Z_{l, i, j}(t+1), C_{l, j}, S_{l, p, j}(t), \beta_{l, p, j}(t), \gamma_{l, p, j}(t)\right)
\end{gathered}
$$

The $\beta_{l, p, j}$ and $\gamma_{l, p, j}$ multipliers will be updated at the end of each iteration using Eqs. 3.7, and 3.8, respectively.

$$
\begin{gathered}
\beta_{l, p, j}(t+1)=\beta_{l, p, j}(t)+\eta\left(C_{l, j}(t+1)-S_{l, p, j}(t+1)\right) \\
\forall j \in\{1, . ., J\}, p \in H_{p}, l \in\{1, . ., L\} \\
\gamma_{l, p, j}(t+1)=\gamma_{l, p, j}(t)+\eta\left(S_{l, p, j}(t+1)-C_{p, j}(t+1)\right) \\
\forall j \in\{1, . ., J\}, p \in H_{p}, l \in\{1, . ., L\}
\end{gathered}
$$

In order to cluster data in a distributed fashion we need to solve the problem in each node. Therefore the sub-problems (Eqs. 3.4, 3.5 and 3.6 ) must be solved in all nodes to find the optimum solution.

\section{Sub-problem 1:}


Because of constraints 3.3.a and 3.3.b on membership matrix $Z$, Eq. 3.4 cannot be solved in a closed-form using a derivation method. Therefore, using the LP methods on Eq. 3.9 to find $\mathrm{Z}$ is required.

$$
Z_{l, i, j}(t+1)=\arg \min _{Z} \sum_{i=1}^{n_{1}} \sum_{j=1}^{J} Z_{l, i, j}\left\|X_{l, j}-C_{l, j}(t)\right\|_{2}^{2}+R \sum_{j=1}^{J}\left|\sum_{i=1}^{n_{l}} Z_{l, i, j} Y_{l, i}\right| \forall l \in\{1, . ., L\}
$$

subject to:

$$
\begin{gathered}
0 \leq Z_{l, i, j} \leq 1, \forall i \in\left\{1, . ., n_{l}\right\}, j \in\{1, . ., J\}, l \in\{1, . ., L\} \\
\sum_{j=1}^{J} Z_{l, i, j}=1, \forall i \in\left\{1, . ., n_{l}\right\}, l \in\{1, . ., L\}
\end{gathered}
$$

Equation 3.9 is exactly the same as Eq. 2.1 in the centralized MagKmeans method in which $C_{l, j}(t)$ represents the centroid of cluster $j$ in node $l$ attained in iteration $t$. It can be re-written as Eq. 3.9.a. The Linear Programming (LP) methods can be used to find the optimal solution.

$$
Z_{l, i, j}(t+1)=\arg \min _{Z, \tau} \sum_{i=1}^{n_{1}} \sum_{j=1}^{J} Z_{l, i, j}\left\|X_{l, j}-C_{l, j}(t)\right\|_{2}^{2}+R \sum_{j=1}^{J} \tau_{l, j} \forall l \in L
$$

subject to:

$$
\begin{gathered}
\tau_{l, j} \geq 0,0 \leq Z_{l, i, j} \leq 1, \forall i \in\left\{1, . ., n_{l}\right\}, j \in\{1, . ., J\}, l \in\{1, . ., L\} \\
\sum_{j=1}^{J} Z_{l, i, j}=1, \forall i \in\left\{1, . ., n_{l}\right\}, l \in\{1, . ., L\} \\
-\tau_{l, j} \leq \sum_{i=1}^{n_{l}} Z_{l, i, j} y_{i} \leq \tau_{l, j}, \forall j \in\{1, . ., J\}, l \in\{1, . ., L\}
\end{gathered}
$$

\section{Sub-problem 2:}

Sub-problem 2 includes finding $S_{l, p, j}$ based on Eq. 3.5. Since there is no constraint left on variable $S$, to solve Eq. 3.5, we can simply try to solve Eq. 3.10.

$$
\frac{\delta F\left(Z_{l, i, j}(t+1), C_{l, j}(t), S_{l, p, j}, \beta_{l, p, j}(t), \gamma_{l, p, j}(t)\right)}{\delta S_{l, p, j}}=0
$$


Therefore:

$$
S_{l, p, j}(t+1)=\frac{1}{2 \eta}\left(\beta_{l, p, j}(t)-\gamma_{l, p, j}(t)\right)+\frac{1}{2}\left(C_{l, j}(t)+C_{p, j}(t)\right)
$$

By substituting $S_{l, p, j}(t+1)$ into Eqs. 3.7 and 3.8:

$$
\begin{aligned}
& \left.\beta_{l, p, j}(t+1)=\frac{1}{2}\left(\beta_{l, p, j}(t)+\gamma_{l, p, j}(t)\right)+\frac{\eta}{2}\left(C_{l, j}(t+1)\right)-C_{p, j}(t+1)\right) \\
& \left.\gamma_{l, p, j}(t+1)=\frac{1}{2}\left(\beta_{l, p, j}(t)+\gamma_{l, p, j}(t)\right)+\frac{\eta}{2}\left(C_{l, j}(t+1)\right)-C_{p, j}(t+1)\right)
\end{aligned}
$$

which means that $\beta_{l, p, j}$ and $\gamma_{l, p, j}$ (the Lagrangian Multipliers) are equal $\forall j \in\{1, . ., J\}, l \in\{1, . ., L\}, p \in$ $H_{l}$. Thus we use $\lambda_{l, p, j}$ to replace both multipliers:

$$
\lambda_{l, p, j}(t+1)=\lambda_{l, p, j}(t)+\frac{\eta}{2}\left(C_{l, j}(t+1)-C_{p, j}(t+1)\right)
$$

\section{Sub-problem 3:}

Following the results of Eq. 3.11, 3.12 and 3.13, the objective function can be rewritten as follows:

$$
\begin{gathered}
F\left(Z_{l, i, j}, C_{l, j}, \lambda_{l, p, j}\right) \\
=\sum_{l=1}^{L} \sum_{i=1}^{n_{l}} \sum_{j=1}^{J} Z_{l, i, j}\left\|X_{l, i}-C_{l, j}\right\|_{2}^{2}+R \sum_{l=1}^{L} \sum_{j=1}^{J}\left|\sum_{i=1}^{n_{l}} Z_{l, i, j} y_{l, i}\right|+\sum_{l=1}^{L} \sum_{j=1}^{J} \sum_{p \in H_{l}} \lambda_{l, p, j}\left(C_{l, j}-C_{p, j}\right) \\
+\frac{\eta}{2} \sum_{l=1}^{L} \sum_{j=1}^{J} \sum_{p \in H_{l}}\left\|C_{l, j}-\frac{1}{2}\left(C_{l, j}(t)+C_{p, j}(t)\right)\right\|^{2} \\
+\left\|\frac{1}{2}\left(C_{l, j}(t)+C_{p, j}(t)\right)-C_{p, j}\right\|^{2}
\end{gathered}
$$

Considering that $\sum_{j=1}^{J} \sum_{p \in H_{l}} C_{l, j} \lambda_{l, p, j}=\sum_{j=1}^{J} \sum_{p \in H_{l}} C_{p, j} \lambda_{p, l, j}, \sum_{p \in H_{l}} \lambda_{l, p, j}=\lambda_{l, j}$ and $\lambda_{l, j}=-\lambda_{j, l}$, Eq. 3.14 can reduce to Eq. 3.15. Then, by solving $\frac{\delta L\left(Z_{l, i, j}(t+1), C_{l, j}, \lambda_{l, p, j}(t)\right)}{\delta C_{l, j}}=0, C_{l, j}(t+1)$ will 
achieve the form given in Eq. 3.16.

$$
\begin{gathered}
F\left(Z_{l, i, j}, C_{l, j}, \lambda_{l, p, j}\right) \\
=\sum_{l=1}^{L} \sum_{i=1}^{n_{l}} \sum_{j=1}^{J} Z_{l, i, j}(t+1)\left\|X_{l, i}-C_{l, j}\right\|_{2}^{2}+R \sum_{l=1}^{L} \sum_{j=1}^{J}\left|\sum_{i=1}^{n_{l}} Z_{l, i, j}(t+1) y_{l, i}\right| \\
+2 \sum_{l=1}^{L} \sum_{j=1}^{J} \lambda_{l, j}(t) C_{l, i}+\eta \sum_{l=1}^{L} \sum_{j=1}^{J} \sum_{p \in H_{l}}\left\|C_{l, j}-\frac{1}{2}\left(C_{l, j}(t)+C_{p, j}(t)\right)\right\|^{2} \\
\left.C_{l, j}(t+1)=\left(\sum_{i=1}^{n_{l}} Z_{l, i, j}(t+1)+\eta\left|H_{l}\right|\right)^{-1}\right)\left[\sum_{n=1}^{n_{l}} Z_{l, i, j}(t+1) X_{l, i}-\lambda_{l, j}(t)\right. \\
\left.+\frac{\eta}{2} \sum_{p \in H_{l}}\left(C_{l, j}(t)+C_{p, j}(t)\right)\right] \\
\lambda_{l, j}(t+1)=\lambda_{l, j}(t)+\frac{\eta}{2} \sum_{p \in H_{l}}\left(C_{l, j}(t+1)-C_{p, j}(t+1)\right)
\end{gathered}
$$

\subsubsection{Proposed Distributed MagKmeans (D-MagKmeans) Algorithm}

Algorithm 3 describes the D-MagKmeans algorithm, which iteratively tries to find the membership matrix $\mathrm{Z}$ using Linear Programming by fixing the cluster centroids at the beginning of each iteration. Figure 3.2 illustrates this algorithm.

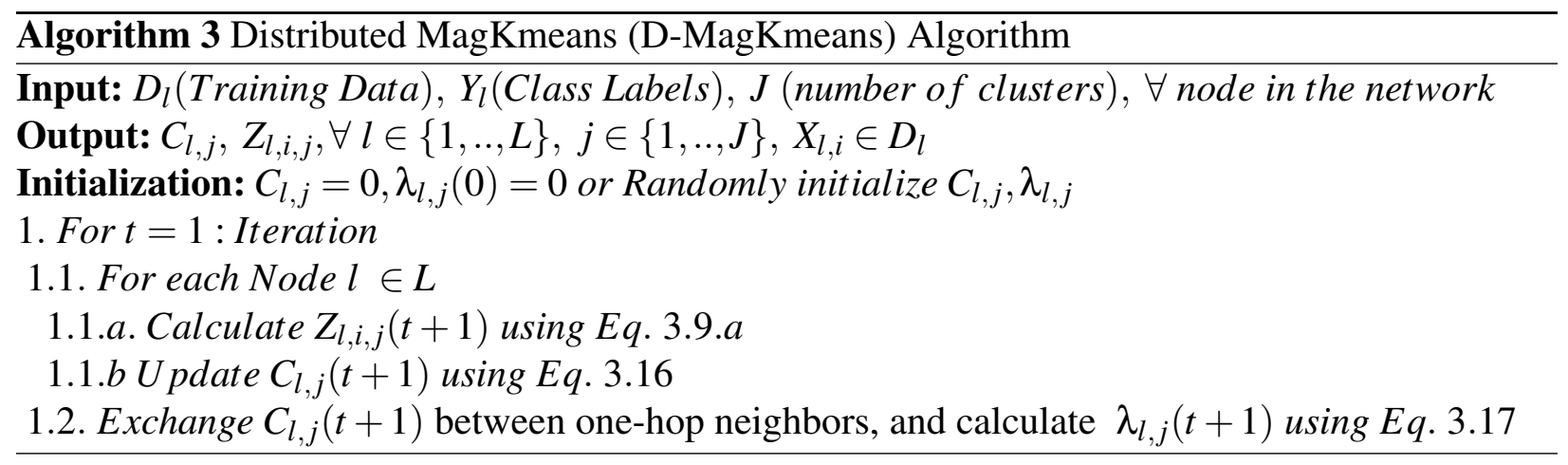

The Lagrangian heuristic multipliers method will converge to an optimum solution, as the 


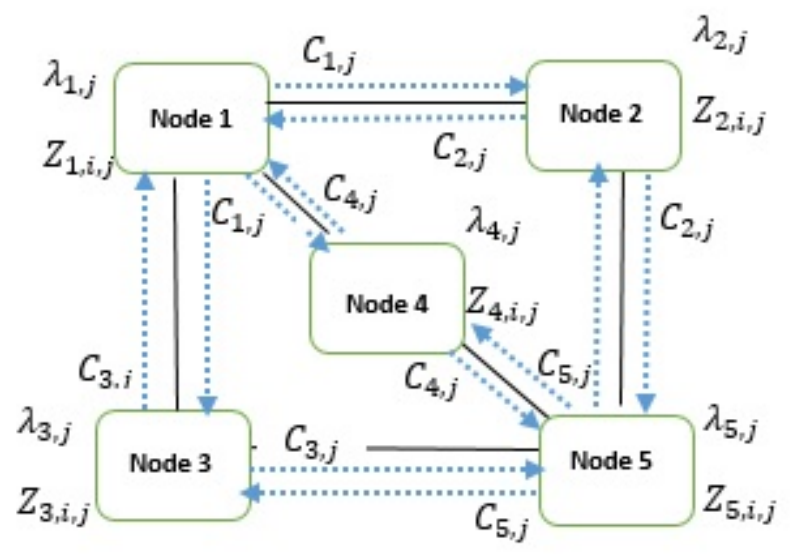

Figure 3.2: A view of D-MagKmeans algorithm

difference in $\lambda_{l, j} \forall j \in\{1, . ., J\}, l \in\{1, . ., L\}$ becomes small. $Z$ is found using Linear Programming with the same objective function as a centralized MagKmeans. The other parameters form a strictly convex objective function with a unique minimizer. Therefore, the proposed algorithm will converge to an optimal solution.

\subsection{Tests and Results}

Fig 3.3 demonstrates how the D-MagKmeans actually works. This figure illustrates the results after applying the D-MagKmeans clustering on a 3-node network connected as shown in Fig 3.4. As illustrated in Fig 3.3, the similar training samples in each node have been categorized in the same cluster as its neighboring node. Each cluster is distinguished by the same color, while the dot (.) represents samples that belong to Class 1 and the cross signs (X) represents samples that belong to Class 2 .

As shown in Fig. 3.3, D-MagKmeans clustered the same number of training data belonging to each class label, in each group. Moreover, data in the close position to each other across the distributed network has been clustered in the same group. 


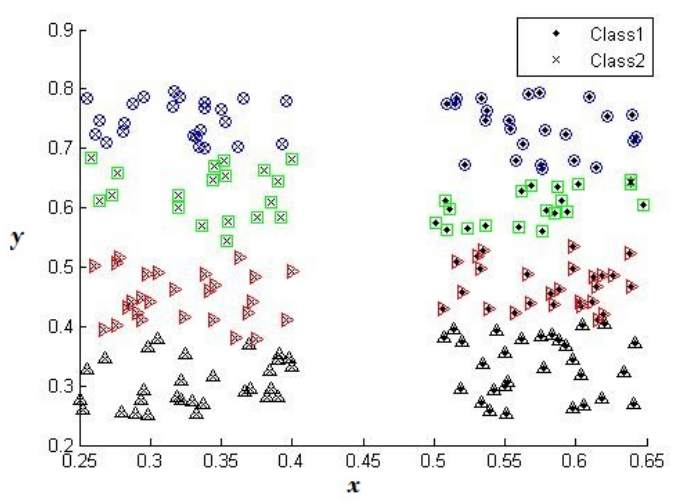

(a) Node 1

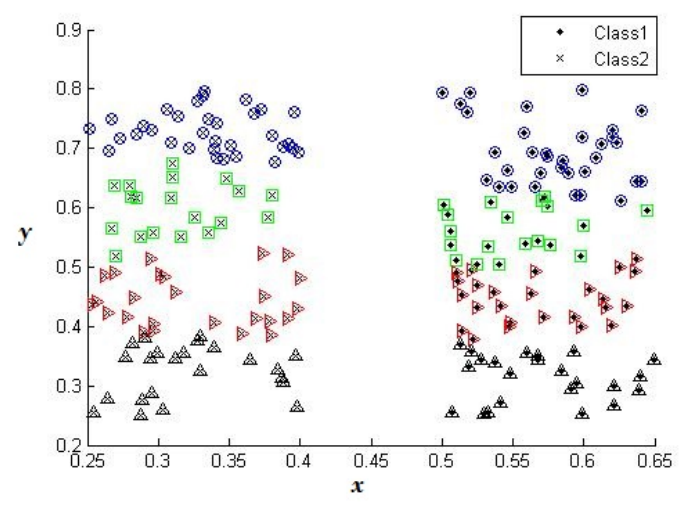

(b) Node 2

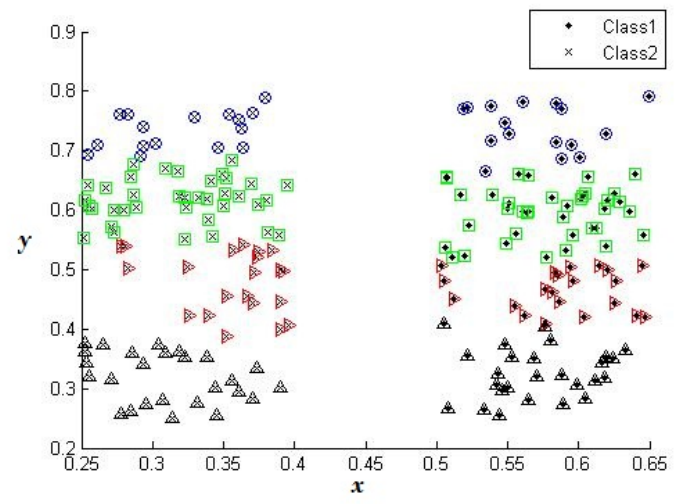

(c) Node 3

Figure 3.3: Results of applying the D-MagKmeans on a 3-node network (each color reprsents one cluster) 


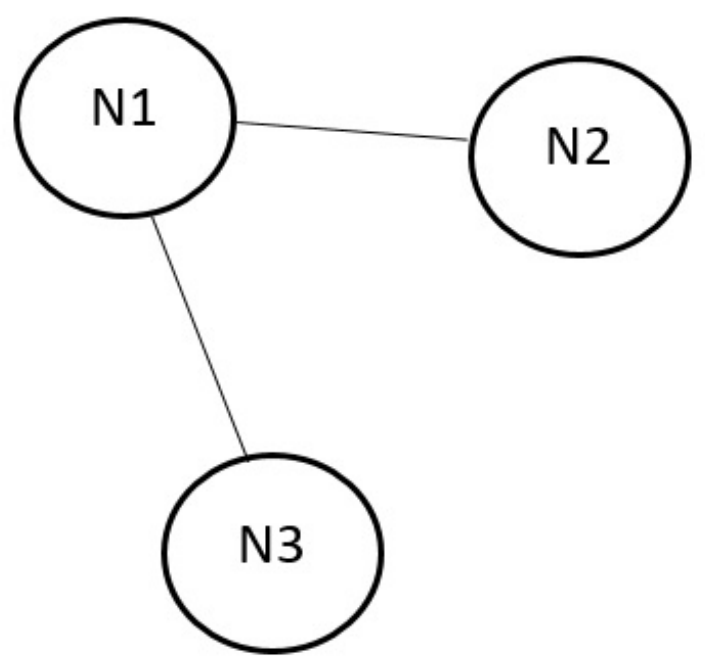

Figure 3.4: A view of a 3-node network connectivity

\subsubsection{Dataset}

Randomly generated data with two different distributions was used. They were labeled into two different classes, Class 1 and Class 2, such that they are not linearly separable. Class 1 , with $\mu=[0,0]$ and $\Sigma=\left[\begin{array}{cc}0.6 & 0 \\ 0 & 0.6\end{array}\right]$ and Class 2 with Multivariate Normal Distribution $\mu_{1}=[-1,-$ 1], $\Sigma_{1}=\left[\begin{array}{cc}0.6 & 0 \\ 0 & 0.6\end{array}\right]$ and $\mu_{2}=[2,2], \Sigma_{2}=\left[\begin{array}{cc}0.6 & 0 \\ 0 & 0.6\end{array}\right]$. D-MagKmeans on a distributed 3-node network, as in Fig 3.4, was performed. The training data includes 50 training samples per class per node. The experiment was run 500 times and the D-Magkmeans algorithm was trained in 100 iterations. Figure 3.5 illustrates the error bars for the D-MagKmeans $(\eta=4)$ and MagKmeans. The results reveal that the performance of the D-MagKmeans is as good as the performance of the centralized one. Regardless of whether all the data are at one node or distributed into several nodes, we still can attain the same clustering results using this approach. 


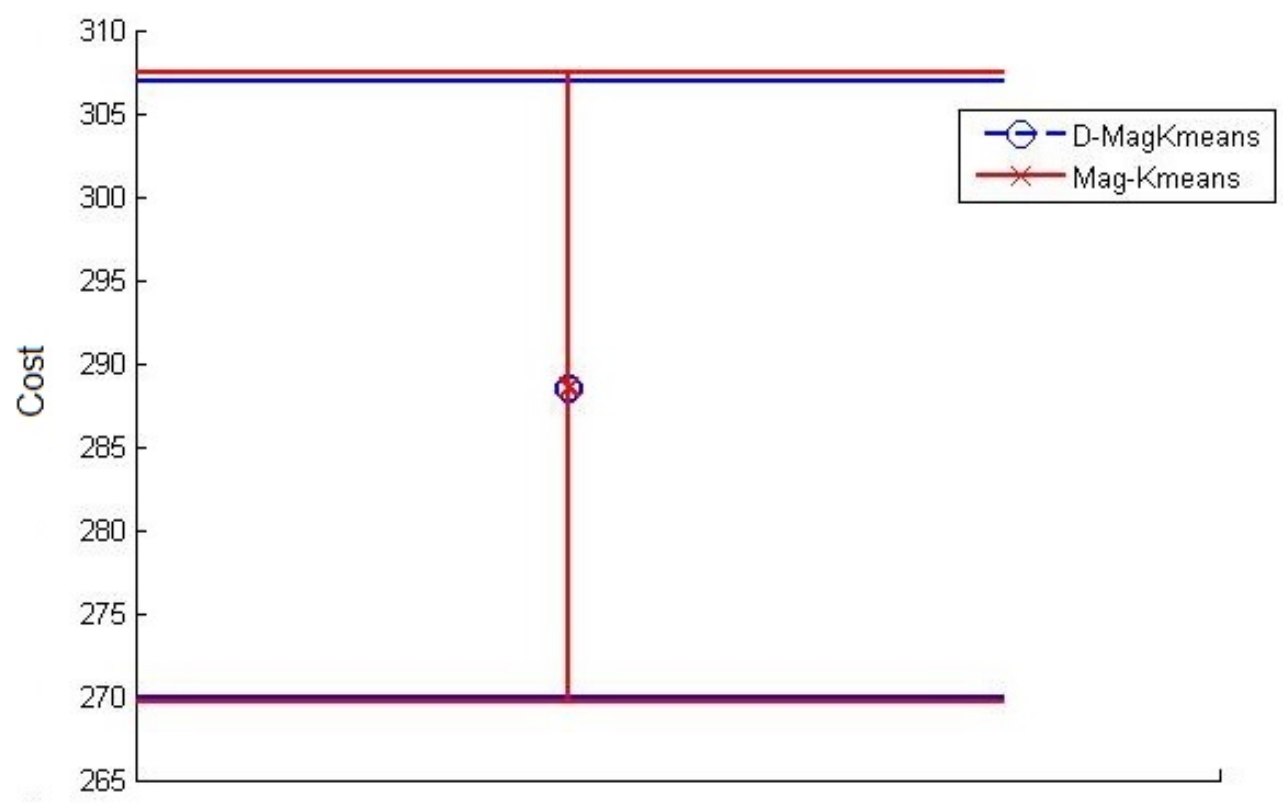

Figure 3.5: Clustering cost comparison of 500 runs each of D-MagKmeans and MagKmeans $(\eta=$ 4)

Table 3.1 shows the Minimum, Mean, and Standard deviation cost of applying the D-MagKmeans and centralized MagKmeans 500 times. Figure 3.6 represents the training process per iteration when $\eta=2,4$, and 10 .

Table 3.1: Cost comparison of D-MagKmeans and centralized MagKmeans

\begin{tabular}{|c|c|c|c|c|c|c|}
\hline \multicolumn{4}{|c|}{ Distributed MagKmeans } & \multicolumn{3}{|c|}{ Centralized MagKmeans } \\
\hline$\eta$ & Min & Mean & STD & Min & Mean & STD \\
\hline 2 & 228.221 & 288.446 & 18.458 & 231.81 & 288.526 & 18.88 \\
\hline 4 & 239.88 & 289.591 & 18.631 & & & \\
\hline 10 & 233.1 & 289.71 & 18.145 & & & \\
\hline
\end{tabular}

Figure 3.7 illustrates the clustering results after applying the D-MagKmeans on the training 


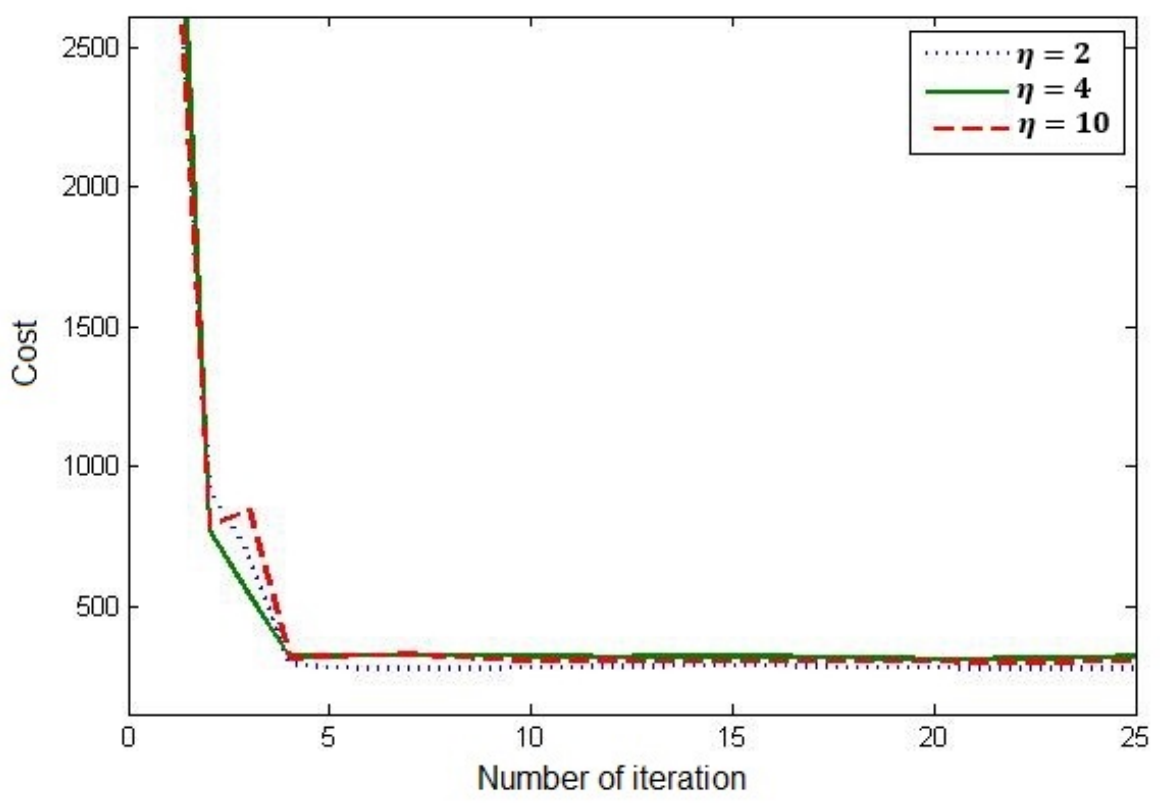

Figure 3.6: Training process of D-MagKmeans for different $\eta$, representing the cost of clustering, per iteration

data with two classes that are not linearly separable. Same clusters are shown in the same color. Data belongs to class 1 are shown with cross sign (X) and class 2 members are shown with dot (.). 


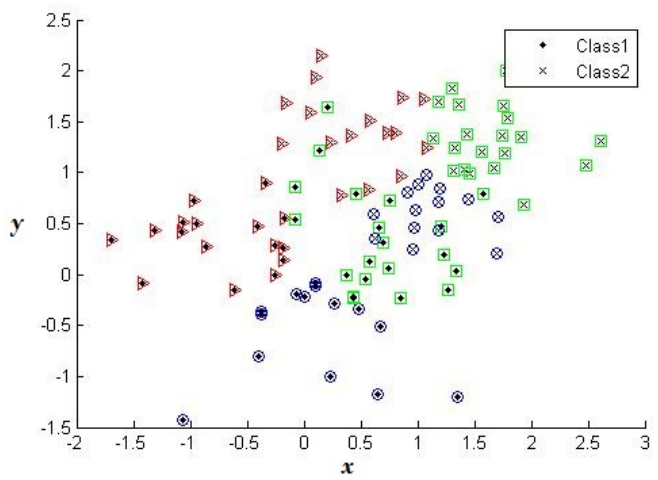

(a) Node 1

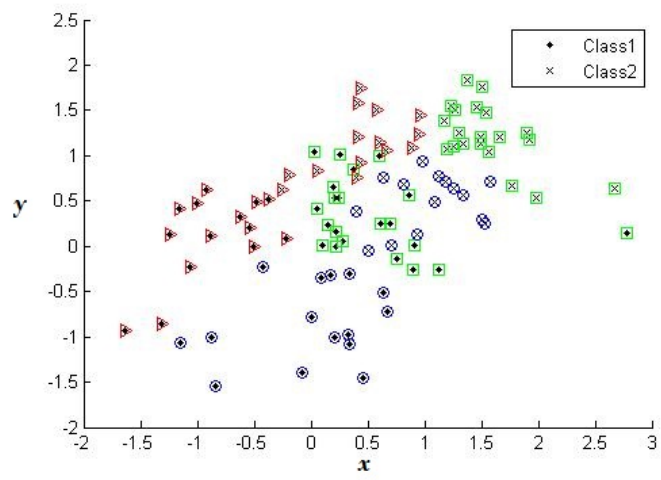

(b) Node 2

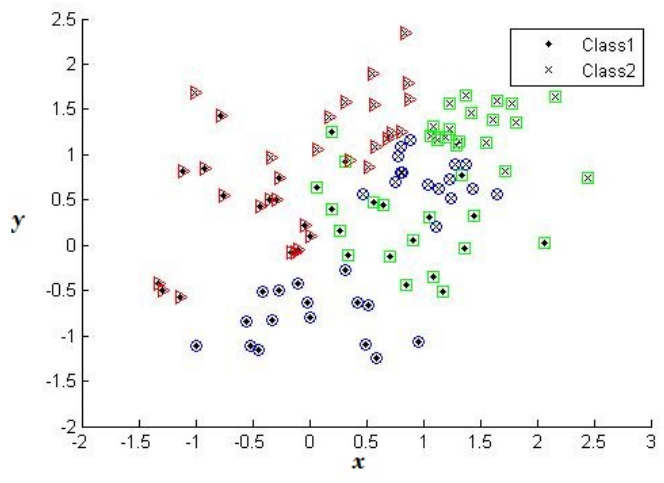

(c) Node 3

Figure 3.7: Clustering results after applying D-MagKmeans

\subsection{Distributed Piece-wise Linear SVM Using D-MagKmeans (D-PWLSVM)}

Using the proposed distributed supervised clustering approach, in this section, we discuss applying the Piece-wise Linear SVM, introduced in Section 2.2, on the result of the clustered data. For a better understanding Fig. 3.8 demonstrates how the proposed approach classifies the data. In this figure, each cluster has its own SVM model achieved by applying a linear SVM on the members of that cluster. 


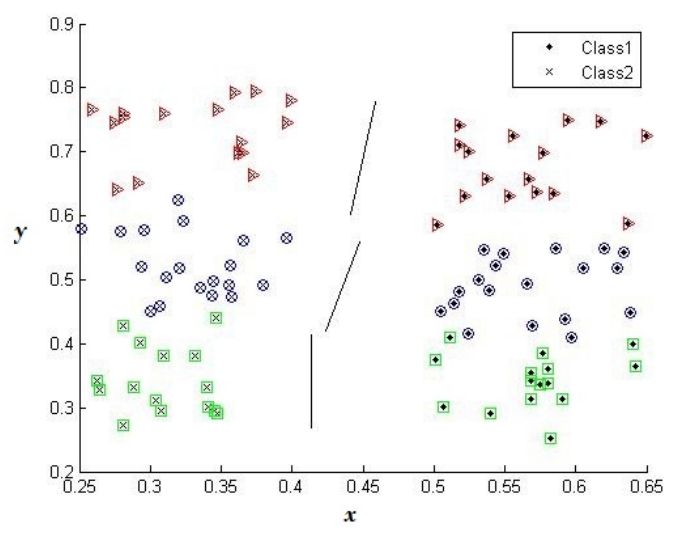

(a) Node 1

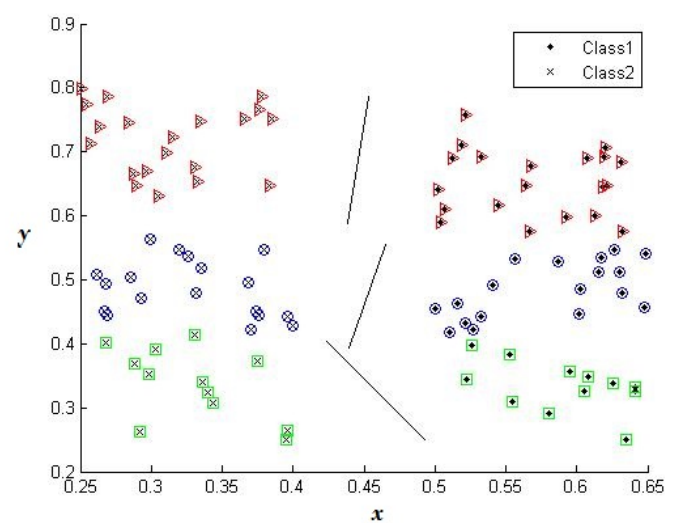

(b) Node 2

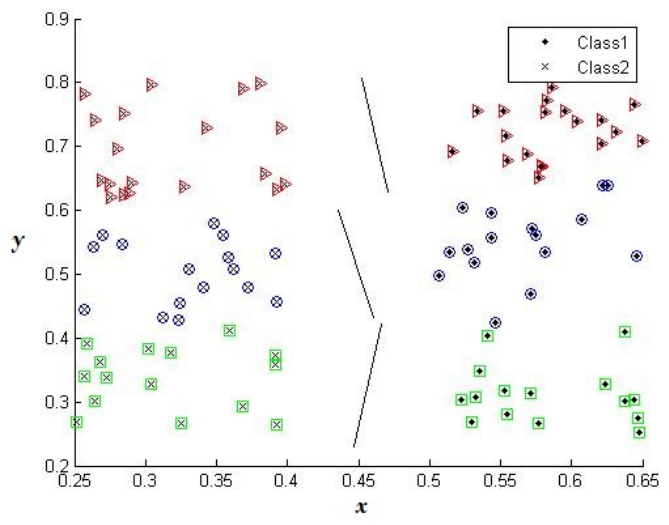

(c) Node 3

Figure 3.8: Simple example of an D-PWLSVM

\subsubsection{Dataset}

This dataset is randomly generated as described in Section 3.2.1. The combined D-PWLSVM approach achieved $82.5 \%$ accuracy, which is comparable to the $86.46 \%$ accuracy achieved by the centralized SVM with a quadratic kernel.

Fig. 3.9 illustrates the SVM decision boundaries output by the D-PWLSVM algorithm. The same clusters can be distinguished with the same color. Data belongs to class 1 and class 2 are shown with dot (.) and cross (x) signs respectively. With $\mathrm{K}=2$, two decision boundaries are built in each nodes. 


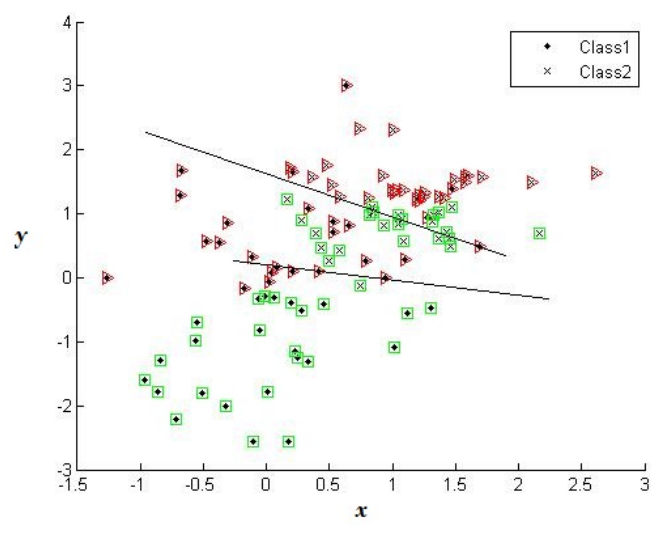

(a) Node 1

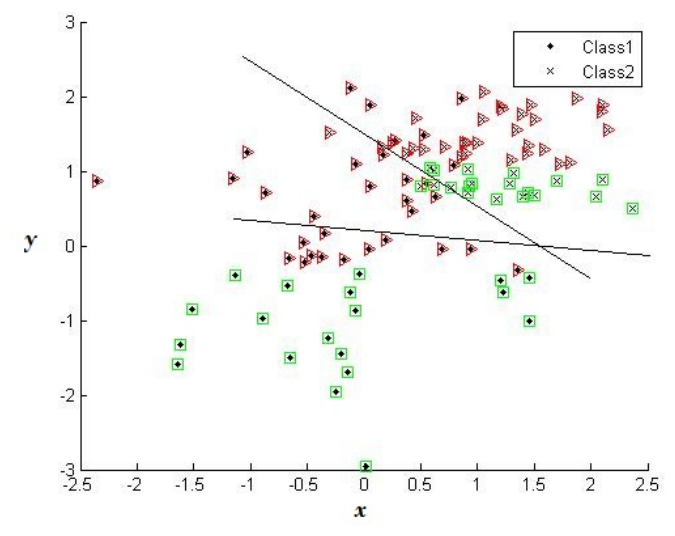

(b) Node 2

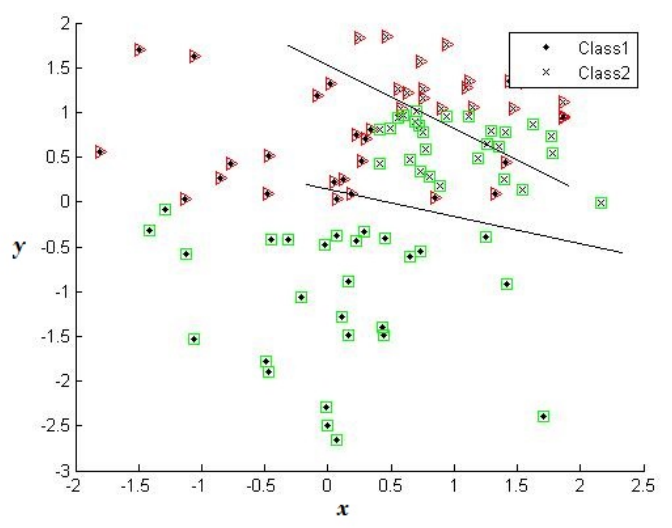

(c) Node 3

Figure 3.9: Results of the D-PWLSVM on randomly generated data

We discuss the efficiency and performance of these approaches in more detail in Chapter 4.

\subsubsection{Summary}

This chapter proposes a new model for the MagKmeans clustering in a distributed environment (D-MagKmeans). The D-PWLSVM is an approach for a distributed classification and is also introduced in this chapter. Algorithm 3 describes the D-MagKmeans algorithm and Fig. 3.2 shows the D-MagKmeans algorithm on a distributed 5-node network example. 


\section{Chapter 4}

\section{Experiments and Results}

This chapter provides the experimental results of the PWLSVM, D-PWLSVM, and Classic SVMs, with different kernels and compares their performance. We present information on the datasets used for validation, their features, and the setups for each of them in Section 4.1. The experimental results and the comparisons between our proposed approaches and the classic SVMs have been discussed in Section 4.2. We summarize the chapter in Section 4.3.

\subsection{Datasets}

The PWLSVM has been employed on four different datasets: Pima Indians Diabetes [3], Waveform [10], Abalone [3] and EHarmony.

\subsubsection{Pima Indians Diabetes Dataset}

This dataset is a publicly available dataset from UCI repository and contains 768 samples with 8 features. Pima Indian Diabetes dataset is used to predict diabetes test results and is divided into 2 classes, class 1 when the patient test result is positive and class 2 otherwise. There are 500 examples of class 1 and 268 of class 2 . We used 450 samples of this dataset equally selected from 
each class.

\subsubsection{Centralized Dataset Setup}

The Piece-wise Linear SVM (PWLSVM) was applied on this dataset 200 times. Each time 250 samples were selected as the training set. To evaluate the algorithm, 200 test samples were chosen randomly from the dataset excluding the training samples.

\subsubsection{Distributed Dataset Setup}

The Distributed Piece-wise Linear (D-PWSVM) was applied on this dataset 200 times. Each time 250 number of training samples were selected randomly and distributed among a 5-node network connected as Fig. 3.2. Each node was assigned an equal number of training samples. To evaluate the algorithm, 200 test samples were chosen from the dataset; this excluded the training samples. The test samples were divided among the nodes equally.

\subsubsection{Waveform Dataset}

The Waveform dataset included 5000 waves with 22 attributes [10]. This dataset is also available on UCI repository. Each attribute had continuous values in the range of $(-5,+5)$. This dataset consists of three classes, each of which generated from a combination of 2 of three base waves. We combined two of these classes and labeled them as class 1 . The other one is labeled as class 2. This thesis focus is mainly on binary classification and it can easily be expanded to multi-class classification. Class 1 includes 3353 samples and class 2 includes 1647 samples. We used 1670 samples of this dataset.

\subsubsection{Centralized Dataset Setup}

SVM with linear, quadratic, RBF, polynomial kernels, and PWLSVM were applied with a maximum iteration of 100,000 to train the SVM. We gathered the results after 200 trials of running 
these algorithms on 1000 randomly selected training data and 670 randomly selected test data.

\subsubsection{Distributed Dataset Setup}

The D-PWSVM was applied on the Waveform dataset 200 times. Each time, 1000 training samples were selected randomly and distributed equally among a 5-node network connected as shown in Fig. 3.2. To evaluate the algorithm, 670 test samples were also chosen. The test samples were divided equally among the nodes.

\subsubsection{Abalone}

This dataset was used to predict the age of abalone from physical measurements. This dataset can be found in the UCI repository. It includes 4177 samples with 8 features divided into 29 classes. Since our focus is on binary classification, the classes have been mapped into two new classes: (1) abalone aged less than 10 years; and (2) abalone aged equal to greater than 10 years.

\subsubsection{Centralized Dataset Setup}

Three thousand training samples and 1160 test samples, including both classes, were equally selected each time. The Linear SVM, RBF, polynomial kernels, and the PWLSVM were used on the Abalone dataset. We also set the maximum iteration of 100,000 for training the SVM. The results were gathered after 200 trials of executing these algorithm.

\subsubsection{Distributed Dataset Setup}

The same number of training and test data as in the centralized setup (3000 and 1160, respectively), were equally distributed among 5 nodes connected as shown in Fig 3.2. The D-PWLSVM algorithm was used on this dataset for 200 trials. 


\subsubsection{EHarmony Dataset}

EHarmony consists of pairs of individuals, which either matched (positive) or did not (negative). This dataset includes 62,548 positive samples and 444,140 negative samples with 116 features. We used 10,000 samples of this dataset equally selected from each class labels.

\subsubsection{Centralized Dataset Setup}

The results were obtained after 200 trials of applying the Piece-wise Linear SVM (PWLSVM) and classic SVM with different kernels on a randomly selected 6000 training data and 4000 test data.

\subsubsection{Distributed Dataset Setup}

The D-PWLSVM was applied on the EHarmony dataset for 200 trials on a 5-node network connected as shown in Fig. 3.2. Each of nodes had 1200 training samples. To evaluate the classifier, 4000 test data were also equally distributed among nodes.

\subsection{Experimental Results}

In this section, the results of applying the proposed methods and the classic SVMs on four different datasets are discussed in detail. In order to measure the required training time in the distributed algorithm (D-PWLSVM), we simply consider the summation of the time for all the steps of all 5 network nodes. The means, minimum, and standard deviation of the errors (percentage) and the training time (seconds) were measured after executing the PWLSVM, D-PWLSVM, and classic SVMs on each dataset. The rest of this section will discuss the results in more detail. 


\subsubsection{Pima Indian Diabetes}

As mentioned previously, the Pima Indian Diabetes dataset is a relatively small dataset of only 768 samples.

Figs. 4.1 and 4.2 illustrate the classifier errors and required training time, respectively, after 200 trials with the number of clusters $(\mathrm{K})$ as 2, 3, 4, and 5. Table 4.1 shows the results in more detail. The D-PWLSVM was run only when $\mathrm{K}=2$ and 3. Since this dataset includes a limited number of samples, dividing them into more than 3 clusters in the distributed algorithm would not likely be very informative.

Table 4.1 illustrates that the small number of data has a degrading effect on the PWLSVM and its distributed method. The classic SVM, on the other hand, outperforms the proposed approach in classifier accuracy and training time.

Table 4.1: Performance comparison (error and training time) between classic SVM, PWLSVM, and D-PWLSVM on the Diabetes dataset

\begin{tabular}{|l|c|c|c|c|c|c|c|c|c|}
\hline & \multicolumn{4}{|c|}{ SVM } & \multicolumn{4}{c|}{ PWLSVM } & \multicolumn{2}{c|}{ D-PWLSVM } \\
\hline & Linear & RBF & Poly & K=2 & K=3 & K=4 & K=5 & K=2 & K=3 \\
\hline Min & 18.50 & 21.00 & 26.5 & 21.00 & 20.00 & 20.00 & 24.00 & 25.5 & 27.00 \\
\hline Mean & 26.20 & 28.59 & 34.95 & 29.00 & 29.52 & 31.28 & 33.08 & 33.30 & 35.22 \\
\hline STD & 2.71 & 2.67 & 3.46 & 2.84 & 3.64 & 3.42 & 3.58 & 3.36 & 3.28 \\
\hline $\begin{array}{l}\text { Average } \\
\text { Time } \\
(\mathrm{sec})\end{array}$ & 0.74 & 0.24 & 3.24 & 0.73 & 0.73 & 0.79 & 0.89 & 0.24 & 0.32 \\
\hline
\end{tabular}




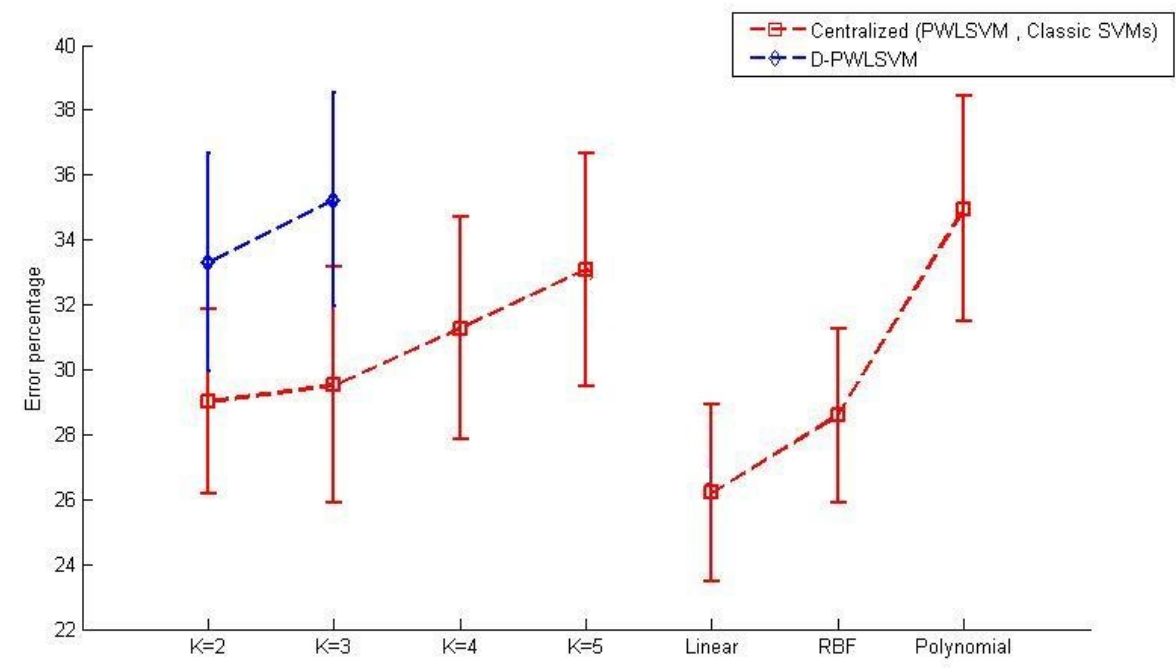

Figure 4.1: Error comparison between D-PWLSVM, PWLSVM, and classic SVM on the Diabetes dataset

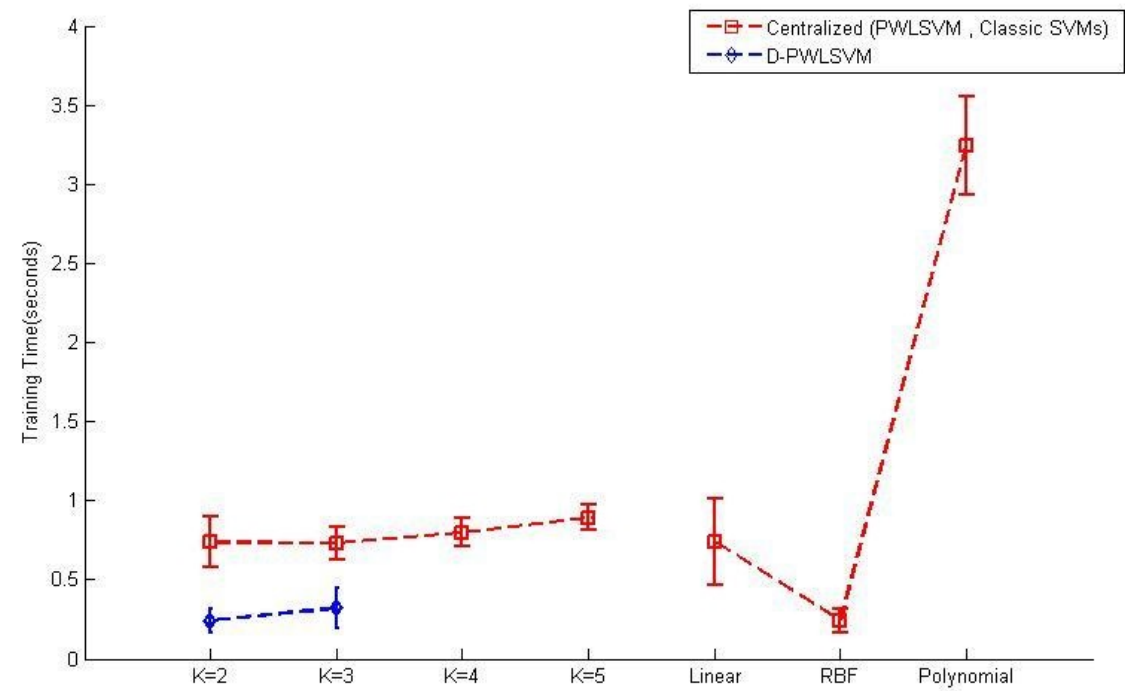

Figure 4.2: Training time comparison between D-PWLSVM, PWLSVM, and classic SVMs on the Diabetes dataset 


\subsubsection{Waveform Dataset}

Figs. 4.3 and 4.4 illustrate the mean and standard deviation of the errors and required training times, respectively, after running the mentioned classifiers on the Waveform dataset. The results of the PWLSVM and D-PWLSVM were calculated with the number of clusters (K) as 2, 3, 4 and 5. Table 4.2 displays the results in more detail.

As implied in Fig. 4.4 and Table 4.2, the PWLSVM $(\mathrm{K}=2)$ and linear SVM perform roughly closely in terms of classification accuracy. However, the PWLSVM outruns the linear SVM 4 minutes faster, which leads to a better classifier when the time trade off is taken into account.

Furthermore, the D-PWLSVM $(\mathrm{K}=2)$ requires 47 seconds less time than the PWLSVM with only $10 \%$ less accuracy. The SVM with RBF kernel converges to a solution in less than a few seconds. However Fig. 4.3 illustrates that the testing error is more than the PWLSVM $(\mathrm{K}=2,3)$ and D-PWLSVM (K=2). Distributed Piece-wise Linear SVM (D-PWLSVM) also makes the classification applicable in distributed networks with privacy concerns.

Table 4.2: Performance comparison (error and training time) between the classic SVM, PWLSVM, and D-PWLSVM on the Waveform dataset

\begin{tabular}{|l|c|c|c|c|c|c|c|c|c|c|c|}
\hline & \multicolumn{4}{|c|}{ SVM } & \multicolumn{4}{c|}{ PWLSVM } & \multicolumn{4}{c|}{ D-PWLSVM } \\
\hline & Linear & RBF & Poly & K=2 & K=3 & K=4 & K=5 & K=2 & K=3 & K=4 & K=5 \\
\hline Min & 7.81 & 12.0 & 9.61 & 6.90 & 9.00 & 12.01 & 13.51 & 12.42 & 14.24 & 15.60 & 15.75 \\
\hline Mean & 10.66 & 17.65 & 12.90 & 10.62 & 11.59 & 18.14 & 19.44 & 17.48 & 19.52 & 21.16 & 22.08 \\
\hline STD & 1.12 & 2.19 & 1.24 & 1.27 & 1.59 & 2.36 & 2.58 & 2.30 & 2.28 & 2.30 & 2.27 \\
\hline $\begin{array}{l}\text { Average } \\
\text { Time } \\
\text { (sec) }\end{array}$ & 291.99 & 0.95 & 346.67 & 48.93 & 39.49 & 18.70 & 17.27 & 1.9970 & 1.3340 & 1.0416 & 0.9119 \\
\hline
\end{tabular}




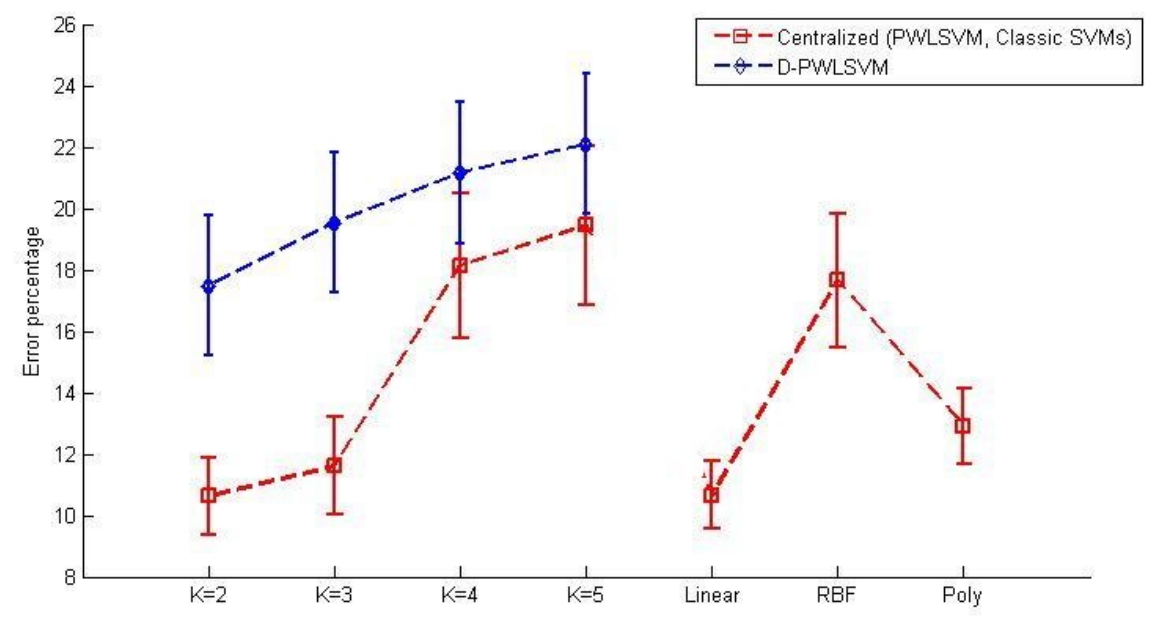

Figure 4.3: Error comparison between the D-PWLSVM, PWLSVM and, classic SVMs on the Waveform dataset

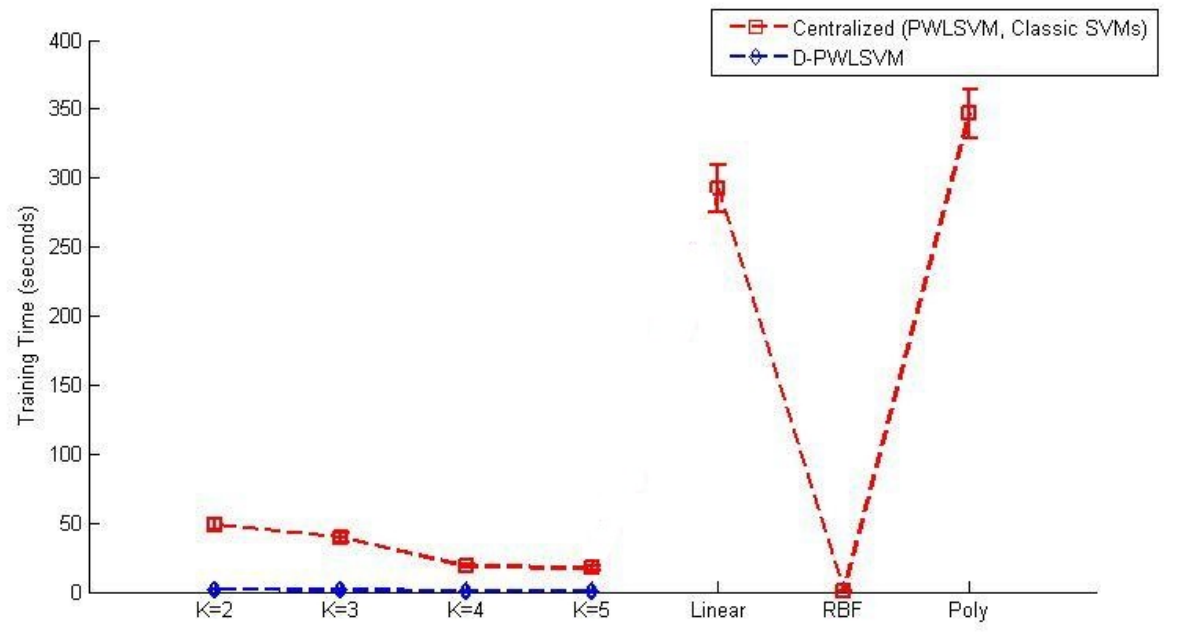

Figure 4.4: Training time comparison between D-PWLSVM, PWLSVM, and classic SVMs on the Waveform dataset 


\subsubsection{Abalone Dataset}

The mean, standard deviation (STD) of errors, and required training times are summarized in Figs. 4.5 and 4.6. These results have been gathered after running the PWLSVM and D-PWLSVM with the number of clusters (K) equal to 2, 3, 4 and 5; and the Classic SVMs with linear, RBF and polynomial kernels on the Abalone dataset. Table 4.3 also shows the results in more detail.

As is illustrated in Table 4.3, PWLSVM $(\mathrm{K}=2)$ has the closest mean to linear SVM. However, it is more robust, due to its smaller standard deviation (STD) and its training time, which is 14 minutes faster than that of the linear SVM.

On the other hand, PWLSVM $(\mathrm{K}=3)$ is less accurate and less robust than when $\mathrm{K}=2$. It is more robust than the linear SVM, whereas its training time is nearly half that of the PWLSVM $(\mathrm{K}=2)$.

Moreover, D-PWLSVM provides a robust result with a significantly less computational expense than the PWLSVM and SVM with Linear, RBF, and Polynomial kernels.

Table 4.3: Comparison of results between the classic SVM, PWLSVM, and D-PWLSVM on the Abalone dataset

\begin{tabular}{|l|c|c|c|c|c|c|c|c|c|c|c|}
\hline & \multicolumn{4}{|c|}{ SVM } & \multicolumn{4}{c|}{ PWLSVM } & \multicolumn{4}{c|}{ D-PWLSVM } \\
\hline & Linear & RBF & Poly & K=2 & K=3 & K=4 & K=5 & K=2 & K=3 & K=4 & K=5 \\
\hline Min & 19.57 & 18.28 & 18.53 & 17.3 & 23.82 & 25.45 & 26.73 & 26.89 & 30.43 & 30.34 & 34.91 \\
\hline Mean & 17.50 & 20.48 & 20.51 & 20.20 & 28.08 & 29.89 & 32.26 & 29.74 & 35.29 & 37.92 & 40.06 \\
\hline STD & 8.63 & 0.94 & 0.98 & 1.25 & 4.56 & 8.90 & 11.75 & 1.34 & 2.18 & 2.34 & 2.34 \\
\hline $\begin{array}{l}\text { Average } \\
\text { Time } \\
\text { (sec) }\end{array}$ & 1556.8 & 1685.5 & 2553.7 & 693.4 & 374.4 & 216.9 & 155.9 & 15.39 & 10.67 & 7.58 & 7.27 \\
\hline
\end{tabular}




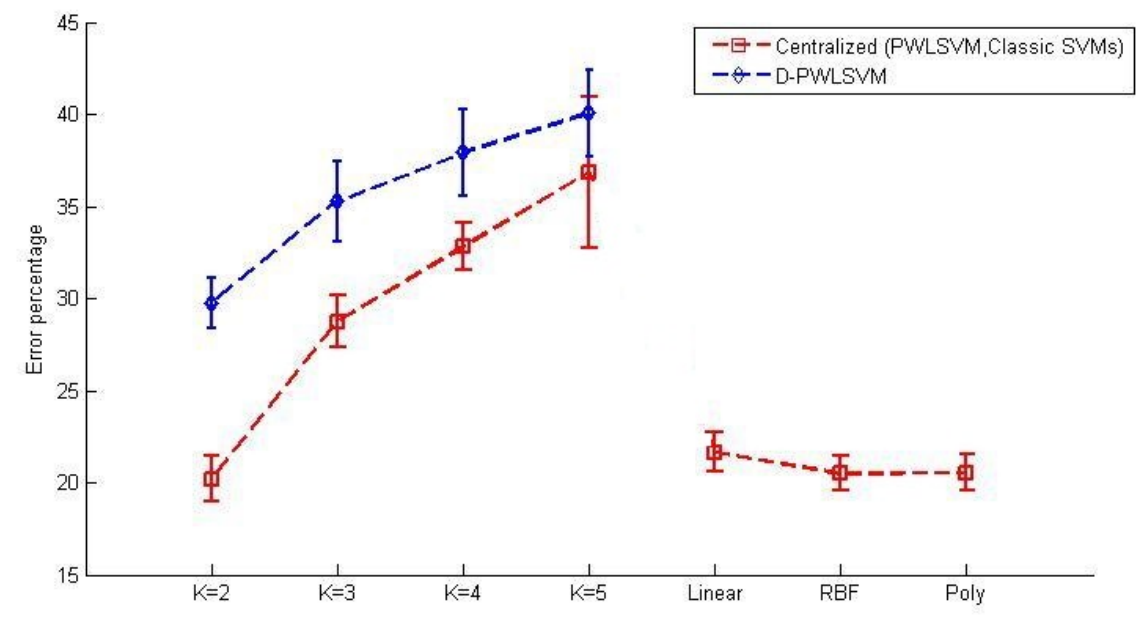

Figure 4.5: Error comparison between the D-PWLSVM, PWLSVM, and the classic SVMs on the Abalone dataset

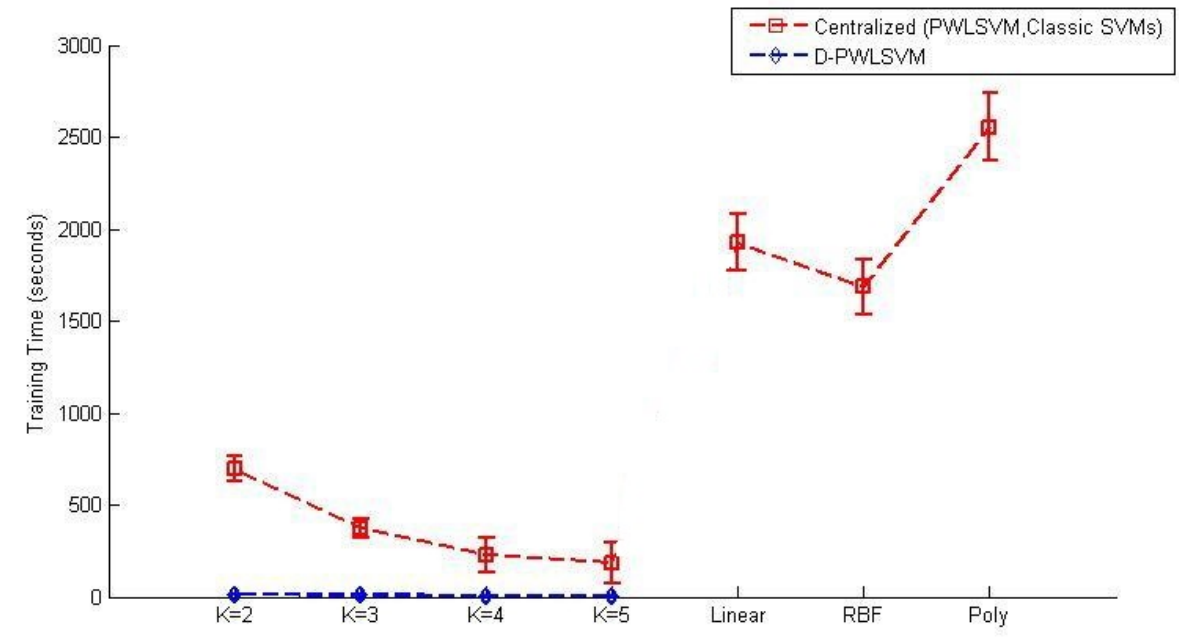

Figure 4.6: Training time comparison between the D-PWLSVM, PWLSVM, and the classic SVMs on the Abalone dataset 


\subsubsection{EHarmony}

Figs. 4.7 and 4.8 illustrate the mean, standard deviation errors and required training times of per-

forming the PWLSVM, D-PWLSVM, and Classic SVMs with linear and RBF kernels. Although we also deployed the SVM with a polynomial kernel on the EHarmony training dataset, it did not converge to a solution within the maximum number of iterations $(100,000)$. The results of the PWLSVM and D-PWLSVM were calculated with the number of clusters $(\mathrm{K})$ of 2, 3, 4 and 5. Table 4.4 presents the detailed results.

PWLSVM $(\mathrm{K}=2)$ and $(\mathrm{K}=3)$ had a close accuracy to the linear SVM, while PWLSVM was almost twice as fast as the linear SVM. On the other hand, the D-PWLSVM (K=2) is almost 12\% less accurate than the linear SVM while its training time was one hour and 23 minutes faster.

D-PWLSVM accuracy is higher than SVM with RBF kernel, however SVM with RBF is faster than D-PWLSVM.

These results reveal a reasonable trade-off that the D-PWLSVM requires when we are dealing with a large quantity of data, memory storage limitation, and distributed network with privacy concerns. 


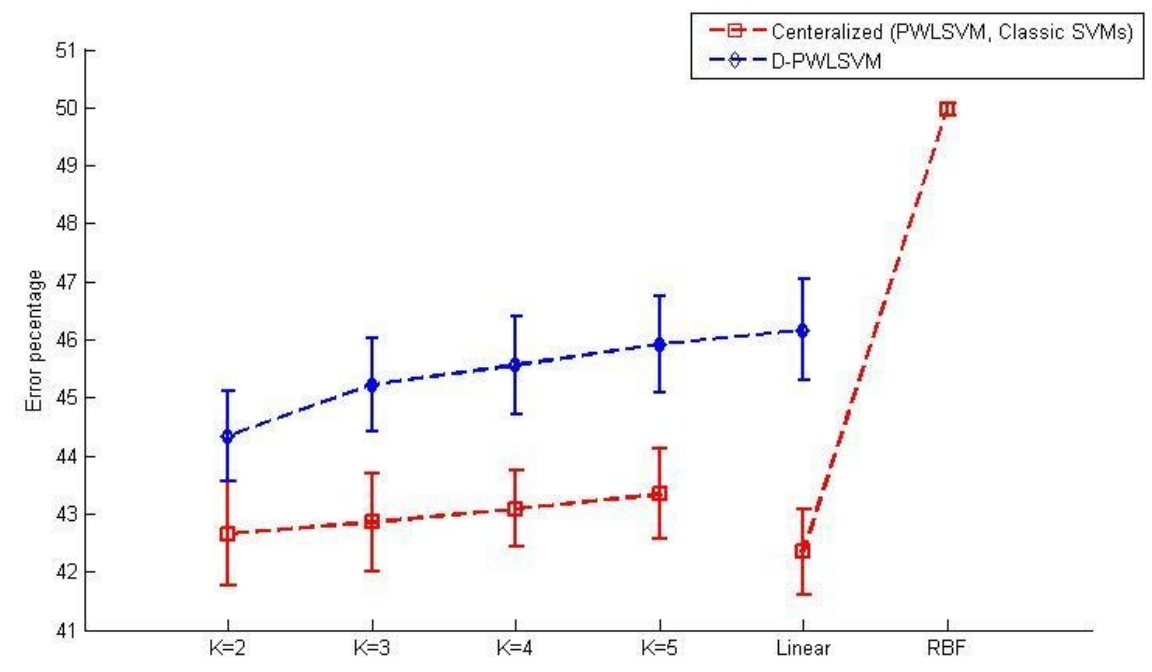

Figure 4.7: Error comparison between D-PWLSVM, PWLSVM, and the classic SVMs on the EHarmony dataset

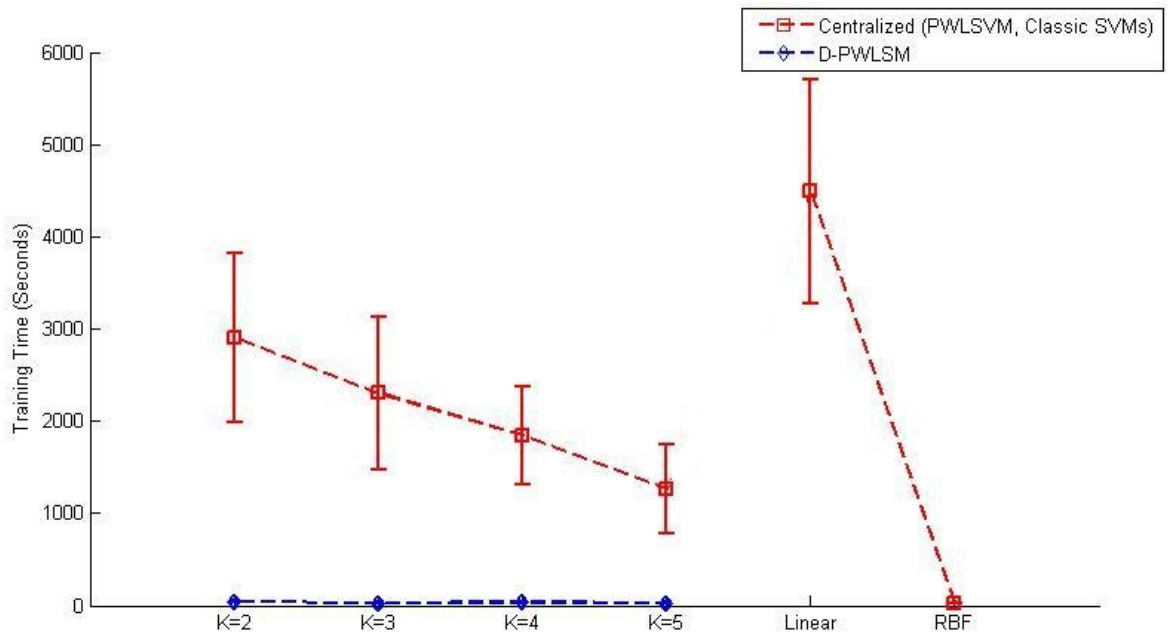

Figure 4.8: Training time comparison between the D-PWLSVM, PWLSVM, and the classic SVMs on the EHarmony dataset 
Table 4.4: Comparison of the results between classic SVM, PWLSVM, and D-PWLSVM on the EHarmony dataset

\begin{tabular}{|l|c|c|c|c|c|c|c|c|c|c|}
\hline & \multicolumn{2}{|c|}{ SVM } & \multicolumn{4}{c|}{ PWLSVM } & \multicolumn{4}{c|}{ D-PWLSVM } \\
\hline & Linear & RBF & $\mathrm{K}=2$ & $\mathrm{~K}=3$ & $\mathrm{~K}=4$ & $\mathrm{~K}=5$ & $\mathrm{~K}=2$ & $\mathrm{~K}=3$ & $\mathrm{~K}=4$ & $\mathrm{~K}=5$ \\
\hline Min & 40.02 & 49.72 & 40.07 & 40.30 & 41.35 & 41.42 & 42.90 & 43.65 & 43.90 & 44.22 \\
\hline Mean & 42.34 & 49.97 & 42.65 & 42.85 & 43.08 & 43.34 & 45.22 & 45.55 & 45.92 & 46.17 \\
\hline STD & 0.75 & 0.11 & 0.91 & 0.85 & 0.65 & 0.77 & 0.80 & 0.83 & 0.82 & 0.88 \\
\hline $\begin{array}{l}\text { Average } \\
\text { Time } \\
\text { (sec) }\end{array}$ & 4488.4 & 27.69 & 2907.8 & 2303 & 1842.3 & 1263.2 & 42.49 & 27.62 & 34.12 & 30.65 \\
\hline
\end{tabular}

\subsection{Summary}

In this chapter we discussed PWLSVM, D-PWLSVM, and classic SVMs on four different datasets: Pima Indian Diabetes, Waveform, Abalone and EHarmony. The results reveal that the PWLSVM, in the centralized environment, can perform better than Classic SVMs, when dealing with large datasets. The D-PWLSVM, outperforms classic SVM with RBF kernel when using EHarmoney. Our distributed approach also is more accurate than SVM with RBF kernel when using Waveform dataset, and is much faster than SVM with RBF kernel when using Abalone dataset. 


\section{Chapter 5}

\section{Conclusion and Future Work}

\subsection{Conclusions}

In classification using the SVM method, the difficulty of finding appropriate kernels and its parameters, the complexity, and the computational cost of mapping data to higher dimension in which training samples cannot be classified linearly, becomes challenging especially when dealing with large datasets.

In this thesis, we introduced a new Piece-wise classification method using the MagKmeans and a linear SVM. Using MagKmeans allowed training data to be preprocessed into fewer groups of smaller numbers of data. Due to the balanced amount of training data in each cluster, which is the result of applying the MagKmeans, our proposed approach was able to classify data faster with very close accuracy to the linear SVM. Experimental results in [30] shows that the linear SVM outperforms the classification with other kernels. The results in Chapter 4 of this thesis show that our proposed approach (PWLSVM) outperforms the classic SVMs.

The results also illustrate that SVM, using RBF, converges to a solution in less than a second. While its classification error is higher than that of the other approaches in most of the cases.

We also proposed a distributed method for the MagKmeans clustering (D-MagKmeans), in 
which training data can be clustered into distributed environments, such as computer networks, by only sending a centroid of clusters to their adjacent computers. Using this approach, the distributed version of the SVM is also introduced. The D-PWLSVM can classify data that are accessible on different computers in a connected network, which performs better than the other methods.

By looking at the results in Chapter 4, it can be seen that the classification accuracy in PWLSVM and D-PWLSVM drops when the training data is divided into more groups. However, the results also show that the training speed increases.

\subsection{Future Work}

In this thesis the focus was mostly on binary classification, the proposed approach can be developed for multiple class classification. Moreover, by using consensus-based linear SVM introduced in [13] instead of linear SVM, we can modify D-PWLSVM to an online learning algorithm. 


\section{Bibliography}

[1] L. Auria and R. A. Moro, "Support vector machines (svm) as a technique for solvency analysis,” Discussion papers//German Institute for Economic Research, Tech. Rep., 2008.

[2] M. Awad, L. Khan, F. Bastani, and I.-L. Yen, "An effective support vector machines (svms) performance using hierarchical clustering," in Tools with Artificial Intelligence, 2004. ICTAI 2004. 16th IEEE International Conference on. IEEE, 2004, pp. 663-667.

[3] C. Blake and C. J. Merz, "Uci repository of machine learning databases [http://www. ics. uci. edu/ mlearn/mlrepository. html]. irvine, ca: University of california," Department of Information and Computer Science, vol. 55, 1998.

[4] D. Boley and D. Cao, "Training support vector machines using adaptive clustering." in SDM. SIAM, 2004, pp. 126-137.

[5] B. E. Boser, I. M. Guyon, and V. N. Vapnik, "A training algorithm for optimal margin classifiers," in Proceedings of the fifth annual workshop on Computational learning theory. ACM, 1992, pp. $144-152$.

[6] M. P. Brown, W. N. Grundy, D. Lin, N. Cristianini, C. Sugnet, M. Ares, and D. Haussler, "Support vector machine classification of microarray gene expression data," University of California, Santa Cruz, Technical Report UCSC-CRL-99-09, 1999. 
[7] C. J. Burges, "A tutorial on support vector machines for pattern recognition," Data mining and knowledge discovery, vol. 2, no. 2, pp. 121-167, 1998.

[8] C. Caragea, D. Caragea, and V. Honavar, "Learning support vector machines from distributed data sources," in PROCEEDINGS OF THE NATIONAL CONFERENCE ON ARTIFICIAL INTELLIGENCE, vol. 20, no. 4. Menlo Park, CA; Cambridge, MA; London; AAAI Press; MIT Press; 1999, 2005, p. 1602.

[9] J. Cervantes, X. Li, and W. Yu, "Support vector machine classification based on fuzzy clustering for large data sets," pp. 572-582, 2006.

[10] C.-C. Chang and C.-J. Lin, "Libsvm: a library for support vector machines, software (2001)."

[11] H. Cheng, P.-N. Tan, and R. Jin, "Efficient algorithm for localized support vector machine," Knowledge and Data Engineering, IEEE Transactions on, vol. 22, no. 4, pp. 537-549, 2010.

[12] S. Del Favero, D. Varagnolo, F. Dinuzzo, L. Schenato, and G. Pillonetto, "On the discardability of data in support vector classification problems," pp. 3210-3215, 2011.

[13] P. A. Forero, A. Cano, and G. B. Giannakis, "Consensus-based distributed support vector machines," The Journal of Machine Learning Research, vol. 11, pp. 1663-1707, 2010.

[14] I. Guyon, B. Boser, and V. Vapnik, "Automatic capacity tuning of very large vc-dimension classifiers," Advances in neural information processing systems, pp. 147-147, 1993.

[15] D. Haussler, "Convolution kernels on discrete structures," Technical report, Department of Computer Science, University of California at Santa Cruz, Tech. Rep., 1999.

[16] M. R. Hestenes, "Multiplier and gradient methods," Journal of optimization theory and applications, vol. 4, no. 5, pp. 303-320, 1969. 
[17] T. Joachims, Text categorization with support vector machines: Learning with many relevant features. Springer, 1998.

[18] A. Navia-Vázquez, D. Gutierrez-Gonzalez, E. Parrado-Hernández, and J. Navarro-Abellan, "Distributed support vector machines," Neural Networks, IEEE Transactions on, vol. 17, no. 4, pp. 1091-1097, 2006.

[19] A. Navia-Vázquez, F. Pérez-Cruz, A. Artes-Rodriguez, and A. R. Figueiras-Vidal, "Weighted least squares training of support vector classifiers leading to compact and adaptive schemes," Neural Networks, IEEE Transactions on, vol. 12, no. 5, pp. 1047-1059, 2001.

[20] E. Parrado-Hernández, I. Mora-Jiménez, J. Arenas-Garcia, A. R. Figueiras-Vidal, and A. Navia-Vázquez, "Growing support vector classifiers with controlled complexity," vol. 36, no. 7. Elsevier, 2003, pp. 1479-1488.

[21] D. Pavlov, J. Mao, and B. Dom, "Scaling-up support vector machines using boosting algorithm," in Pattern Recognition, 2000. Proceedings. 15th International Conference on, vol. 2. IEEE, 2000, pp. 219-222.

[22] J. C. Platt, "Fast training of support vector machines using sequential minimal optimization," in Advances in kernel methods. MIT press, 1999, pp. 185-208.

[23] B. Schölkopf, A. Smola, and K.-R. Müller, "Kernel principal component analysis," in Artificial Neural NetworksICANN’97. Springer, 1997, pp. 583-588.

[24] B. Schölkopf and A. J. Smola, Learning with kernels: support vector machines, regularization, optimization, and beyond. MIT press, 2002.

[25] A. Shilton, M. Palaniswami, D. Ralph, and A. C. Tsoi, "Incremental training of support vector machines," Neural Networks, IEEE Transactions on, vol. 16, no. 1, pp. 114-131, 2005. 
[26] N. A. Syed, S. Huan, L. Kah, and K. Sung, "Incremental learning with support vector machines," 1999.

[27] V. Vapnik, S. E. Golowich, and A. Smola, "Support vector method for function approximation, regression estimation, and signal processing," Advances in neural information processing systems, pp. 281-287, 1997.

[28] Y.-M. Wen and B.-L. Lu, "A cascade method for reducing training time and the number of support vectors," in Advances in Neural Networks-ISNN 2004. Springer, 2004, pp. 480-486.

[29] H. Yu, J. Yang, and J. Han, "Classifying large data sets using svms with hierarchical clusters," in Proceedings of the ninth ACM SIGKDD international conference on Knowledge discovery and data mining. ACM, 2003, pp. 306-315.

[30] G.-X. Yuan, C.-H. Ho, and C.-J. Lin, "Recent advances of large-scale linear classification," Proceedings of the IEEE, vol. 100, no. 9, pp. 2584-2603, 2012.

[31] Y. Zhan and D. Shen, "Design efficient support vector machine for fast classification," Pattern Recognition, vol. 38, no. 1, pp. 157-161, 2005. 